\title{
Radio Detection of Colliding Wind Binaries
}

\author{
Paula Benaglia ${ }^{\mathrm{A}, \mathrm{B}, \mathrm{E}}$, Bärbel Koribalski ${ }^{\mathrm{C}}$, and Juan F. Albacete Colombo ${ }^{\mathrm{B}, \mathrm{D}}$ \\ A Instituto Argentino de Radioastronomía, C.C. 5, Villa Elisa (1894), Argentina \\ ${ }^{B}$ Facultad de Ciencias Astronómicas y Geofísicas, UNLP, 1900 La Plata, Argentina \\ C Australia Telescope National Facility, CSIRO, Epping NSW 1710, Australia \\ D Osservatorio Astronomico di Palermo, 90134 Palermo, Italy \\ E Corresponding author. Email: pbenaglia@fcaglp.unlp.edu.ar
}

Received 2005 November 16, accepted 2006 January 30

\begin{abstract}
Four massive, early-type stars, three of which are confirmed binaries, have been observed with the Australia Telescope Compact Array at 1.4, 2.4, 4.8, and 8.6 GHz. The earliest star cataloged so far, HD 93129A, was also observed at 17.8 and $24.5 \mathrm{GHz}$. Here we present an analysis of the spectra as well as the structure of the stellar systems. All four spectra show clear evidence of non-thermal emission, indicative of a binary system with a colliding wind region. We discuss the magnetic field of the emitting region of HD 93129A and make predictions on the radiation at high energies. Archive X-ray observations towards the target sources are also investigated and interpreted in the light of the non-thermal emission detected.
\end{abstract}

Keywords: stars: early-type — stars: individual (CD-47 4551, HD 93129A, HD 124314, HD 150136) — stars: mass loss — stars: winds, outflows — radio continuum: stars

\section{Introduction}

Through supersonic winds massive, early-type stars (OB to Wolf-Rayet) emit particles as well radiation into the circumstellar medium. Spectroscopic measurements show that stellar wind velocities reach thousands of kilometres per second. The fact that these velocities are larger than the stellar escape velocity proves that important stellar mass loss takes place, especially during and after the end of the main sequence life phase. Mass loss affects the evolution of the star.

The plasma forming the wind produces a thermal excess in flux density from the infrared to the radio range, detectable — and detected - by telescopes with high sensitivity and angular resolution (see the comprehensive observational work by Bieging, Abbott, \& Churchwell 1989, for example). However, there are numerous stars that show evidence for non-thermal (NT) emission, revealing the presence of relativistic electrons tangled in the stellar magnetic fields. Local instabilities (e.g., Lucy \& White 1980) or magnetic confinement (Jardine et al. 2001) can give rise to particle acceleration in single winds. In a stellar system composed of two stars with interacting winds, relativistic particles are expected to be produced in the region where both winds interact (Eichler \& Usov 1993).

In general, early-type stars are X-ray emitters, with a characteristic thermal spectrum, X-ray temperatures of $\geq 1 \mathrm{keV}$, and $\mathrm{X}$-ray luminosities of about $10^{-7} L_{\mathrm{bol}}$ (Berghöfer et al. 1997). In colliding wind binaries, the shock-heated gas at the colliding wind region (CWR) will bring about an enhancement of X-ray emission, which can be considered as a signature of the existence of winds interacting. However, the thermal and/or non-thermal origin of the X-ray radiation strictly depends on the physical parameters and configuration of the stellar system. In close binary systems, the CWR is embedded in the wind acceleration zone: The shocks are less energetic compared to those in wide systems - and there is thermal radiation mostly at soft X-rays. On the contrary, wide binaries can have strongly colliding winds that produce thermal emission above $3 \mathrm{keV}$ and are capable of forming the $\mathrm{Fe}_{\mathrm{K} \alpha} 6.7 \mathrm{keV}$ emission line, as well as non-thermal emission from the inverse Compton (IC) scattering.

From an observational point of view, the existence of non-thermal X-ray emission does not imply the existence of non-thermal radio emission, because the radio emission forms deep inside the wind where it would be strongly absorbed. However, the inverse situation would be interesting to discuss.

As searches for early-type companions to early-type stars advance, there is diminishing evidence for single stars with non-thermal radio emission. An example is $9 \mathrm{Sgr}$, an $\mathrm{O} 4 \mathrm{~V}((\mathrm{f}+))$ star, often used to test radio emission models of NT emission from single stars, and very recently re-discovered as a binary with an early-type companion (Rauw et al. 2005). Another rare object is HD 168112, for which radio and X-ray observations show strong evidence of a binary system whereas optical observations have been unable to discover a companion (Blomme et al. 2005; De Becker et al. 2004; Rauw et al. 2005).

In a few cases, the CWR has been identified through radio interferometric observations (e.g. WR 146, Dougherty et al. 1996; WR 147, Moran et al. 1989; Cyg OB2 no. 5, Contreras et al. 1997; WR 140, Dougherty et al. 2005). For 
Table 1. Target sources

\begin{tabular}{|c|c|c|c|c|c|c|}
\hline Name & RA,Dec (J2000) [hms, dms] & Sp. type & $d[\mathrm{kpc}]$ & $\log (\dot{M})$ & $v_{\infty}\left[\mathrm{km} \mathrm{s}^{-1}\right]$ & Status \\
\hline $\mathrm{CD}-47^{\circ} 4551$ & $085754.620-474415.73$ & $\mathrm{O} 4 \mathrm{III}(\mathrm{f})^{\mathrm{A}}$ & $1.3^{\mathrm{B}}$ & -5.38 & {$[3000]^{\mathrm{C}}$} & $?$ \\
\hline HD 93129A & $104357.462-593251.27$ & O2 If ${ }^{* D}$ & $2.5^{\mathrm{E}}$ & -4.58 & $3200^{\mathrm{F}}$ & Binary $^{\mathrm{G}}$ \\
\hline HD 124314 & $141501.605-614224.39$ & O6 V $(n)((f))^{\mathrm{H}}$ & $1.0^{\mathrm{B}}$ & -6.15 & $2500^{\mathrm{I}}$ & Binary ${ }^{\mathrm{D}}$ \\
\hline HD 150136 & $164120.415-484546.64$ & $03.5 \mathrm{If}^{* J}$ & $1.3^{\mathrm{K}}$ & -5.24 & $3160^{\mathrm{I}}$ & Multiple $^{\mathrm{J}}$ \\
\hline
\end{tabular}

${ }^{\mathrm{A}}$ Walborn (1982). ${ }^{\mathrm{B}}$ See text. ${ }^{\mathrm{C}}$ Interpolated from Prinja et al. (1990). ${ }^{\mathrm{D}}$ Walborn et al. (2002). ${ }^{\mathrm{E}}$ Walborn (1995). ${ }^{\mathrm{F}}$ Taresch et al. (1997).

${ }^{\mathrm{G}}$ Nelan et al. (2004). ${ }^{\mathrm{H}}$ Walborn (1973). ${ }^{\mathrm{I}}$ Howarth et al. (1997). ${ }^{\mathrm{J}}$ Niemela \& Gamen (2005). ${ }^{\mathrm{K}}$ Herbst \& Havlen (1977).

WR 146 and WR 147, theoretical calculations of the spatial and spectral distribution of the radio emission were tested against radio data (Dougherty et al. 2003; O'Connor et al. 2005), yielding a good agreement.

For single early-type stars, theoreticians find some diffculties in reproducing the slow decrease in emissivity inside the wind, with the distance to the star, although the latest models involve layered synchrotron-emitting regions and the relevant cooling mechanisms (van Loo 2005).

As part of a survey of radio emission from massive, early-type stars, we have performed continuum observations of the brightest, nearest, and earliest undetected southern OB stars at 8.6 and 4.8 GHz (Benaglia et al. 2001; Benaglia \& Koribalski 2004). Four of the observed stars displayed negative spectral indices $(\alpha<0)$, suggesting a significant non-thermal contribution to their radio emission. We use $S_{v} \propto v^{\alpha}$, where $S_{v}$ is the flux density at frequency $v$ and $\alpha$ is the spectral index. To further study the radio emission from these stars we also observed them at lower frequencies. Here we present 2.4 and $1.4 \mathrm{GHz}$ observations obtained with the Australia Telescope Compact Array $\left(\mathrm{ATCA}^{1}\right.$ ) for the stars CD-47 ${ }^{\circ}$ 4551, HD 93129A, HD 124314, and HD 150136. Preliminary results have been presented in Benaglia \& Koribalski (2006). Based on the presence of non-thermal radio emission, presumably coming from the CWR of a binary star, we looked for other indicators of binarity. We complemented our analysis of the radio results with a study of all archive X-ray observations towards the stars.

The organization of the paper is as follows. Section 2 gathers the relevant stellar information. Section 3 explains the observing technique and reduction strategy. Section 4 briefly sums up different mechanisms contributing to the emission from the stars in the radio domain. The radio results are given in Section 5. In Section 6 we analyze the available X-ray data towards the target fields. We discuss the scenario for each object in Section 7, and Section 8 closes with our conclusions.

\section{Selected Stars}

The stars were selected from the targets already observed at 3 and $6 \mathrm{~cm}(8.64$ and $4.8 \mathrm{GHz}$, Benaglia et al. 2001; Benaglia \& Koribalski 2004) that showed a non-thermal contribution to the emission. In order to investigate where

\footnotetext{
${ }^{1}$ The Australia Telescope is funded by the Commonwealth of Australia for operation as a National Facility managed by CSIRO.
}

the radio emission is coming from, it is fundamental to know the binary status of the stars, and the structure of the systems.

The parameters adopted for the present target stars are listed in Tables 1 and 2. The equatorial coordinates were taken as quoted in the GOS Catalogue (Maíz-Apellániz et al. 2004). The references for the spectral types and wind terminal velocities are given in Table 1 . The distances to CD-47 4551 and HD 124314 are spectro-photometric ones, derived from the apparent magnitude and $(B-V)$ colour index quoted as in Benaglia et al. (2001), intrinsic colour indices from Wegner (1994), and absolute magnitudes from Martins, Schaerer, \& Hillier (2005). The mean molecular weight of the ions $(\mu)$ was assumed 1.5 for the earliest types, and 1.3 for the later ones. For the HD 93129A system, the mass loss rate $(\dot{M})$ was taken from Repolust, Puls, \& Herrero (2004). The $\dot{M}$ values for the rest of the stars were computed by means of the mass-loss rate recipe given by Vink, de Koter, \& Lamers $(2000)^{2}$. The effective temperatures $\left(T_{\text {eff }}\right)$, stellar masses $\left(M_{*}\right)$, and stellar luminosities $\left(L_{*}\right)$ are from the calibration tables of Martins, Schaerer, \& Hillier (2005), obtained with observational $T_{\text {eff }}$ scales.

\section{$2.1 C D-47^{\circ} 4551$}

Also known as CPD-47 2963 , CD- $47^{\circ} 4551$ is the least studied object of the group. Walborn (1982) catalogued it as an O4 III(f). Its binary status is still unknown. Photometric measurements done at CASLEO (San Juan, Argentina), show the absence of another early-type, massive visual companion (P. Ostrov 2001, private communication). Spectroscopic optical observations are currently under way to clarify this issue.

Benaglia et al. (2001) detected CD-47 4551 with ATCA observing simultaneously at 3 and $6 \mathrm{~cm}$ over $160 \mathrm{~min}$. The measured flux densities were $S_{3 \mathrm{~cm}}=1.77 \pm 0.05 \mathrm{mJy}$ and $S_{6 \mathrm{~cm}}=2.98 \pm 0.05 \mathrm{mJy}$. The corresponding spectral index is $\alpha=-0.89 \pm 0.06$.

\section{$2.2 H D 93129 A$}

This is one of the earliest $\mathrm{O}$ stars cataloged so far, as O2 If* (Walborn et al. 2002). It belongs to the cluster Trumpler 14, in the Carina region, at $2.5 \mathrm{kpc}$ (Walborn 1995). By means of HST observations Nelan et al. (2004) found the star has an OB companion, 55 mas away (or $\approx 140 \mathrm{AU}$ at $2.5 \mathrm{kpc}$ ). The companion is assumed coeval and of the same spectral type of various other $\mathrm{OB}$ stars nearby $(\mathrm{O} 3.5 \mathrm{~V})$. Recent

\footnotetext{
2 astro.ic.ac.uk/jvink
} 
Table 2. Adopted parameters

\begin{tabular}{rcccc}
\hline Name & $T_{\text {eff }}[\mathrm{K}]$ & $\log \left(L_{*}\right)\left[\mathrm{L}_{\odot}\right]$ & $M_{*}\left[\mathrm{M}_{\odot}\right]$ & $\mu$ \\
\hline $\mathrm{CD}-47^{\circ} 4551$ & $42422^{\mathrm{A}}$ & $5.85^{\mathrm{A}}$ & $47^{\mathrm{A}}$ & 1.5 \\
HD 93129A & $42500^{\mathrm{B}}$ & $6.17^{\mathrm{B}}$ & $95^{\mathrm{B}}$ & 1.5 \\
HD 124314 & $38867^{\mathrm{A}}$ & $5.32^{\mathrm{A}}$ & $31^{\mathrm{A}}$ & 1.3 \\
HD 150136 & & & & \\
'A' & $41300^{\mathrm{A}}$ & $5.96^{\mathrm{A}}$ & $62^{\mathrm{A}}$ & 1.5 \\
'B' & $38867^{\mathrm{A}}$ & $5.32^{\mathrm{A}}$ & $31^{\mathrm{A}}$ & 1.3 \\
\hline
\end{tabular}

${ }^{\text {A }}$ Martins et al. (2005). ${ }^{\text {B }}$ Repolust et al. (2004).

values of stellar luminosity, mass, and effective temperature for the system were derived by Repolust, Puls, \& Herrero (2004) in their models taking into account line-blocking/ blanketing effects.

At $2.6^{\prime \prime}$ (6500 AU at $2.5 \mathrm{kpc}$ ) from HD 93129A is the O3.5 V star HD 93129B. A hard X-ray source coincident with the position of the stars was detected by Chandra (Evans et al. 2003, 2004).

The Tr 14 field was observed with the ATCA at 3 and $6 \mathrm{~cm}$ (Benaglia \& Koribalski 2004), and HD 93129A was the only detection. The measured flux densities were $S_{3 \mathrm{~cm}}=2.0 \pm$ $0.2 \mathrm{mJy}$ and $S_{6 \mathrm{~cm}}=4.1 \pm 0.4 \mathrm{mJy}(\alpha=-1.2 \pm 0.3)$.

\section{$2.3 H D 124314$}

This target has been classified as an O6 V(n)((f)) by Walborn (1973). Feast, Thackeray, \& Wesselink (1954) proposed it as a possible spectroscopic binary ('SB1?'), and it has a visual companion $2.7^{\prime \prime}$ away (about $2700 \mathrm{AU}$ at 1 $\mathrm{kpc}$ ). This star appears as the main ionizing agent of the HII region RCW 85 (Yamaguchi et al. 1999). It has counterparts at other wavelengths - in the X-ray the ROSAT source 1RXS J141500.1-614231 and in the IR the IRAS source $14111-6127$ — and it is superposed to the probability contours of the gamma-ray source 3EG J1410-6147. The study of de Wit et al. (2004) confirms it is a field star.

The star was observed in three opportunities with ATCA at 3 and $6 \mathrm{~cm}$, with flux density variations (see Benaglia et al. 2001, and Table 3).

\section{$2.4 H D 150136$}

This star is a member of the open cluster NGC 6193, in the Ara OB1 association, at a distance of $1.3 \mathrm{kpc}$ (Herbst \& Havlen 1977). It is the brightest star of a multiple system, with the closest visual component 1.6 arcsec apart, called MIO 8 AB (see Mason et al. 1998, and references therein). The second brightest star in the group is HD 150135, at about 10 arcsec. Very recently, an optical study by Niemela \& Gamen (2005), allowed them to re-classify HD 150136 as an O3.5 If* (HD 150136A), to confirm a second close O6 V companion (HD 150136B) with a period of 2.7 days, and suggest a third close OB-type component. With these data available a picture of the system can be sketched. For a circular orbit of HD 150136A/B, the separation would be about $40 \mathrm{R}_{\odot}$ at $1.3 \mathrm{kpc}$. The visual companion MIO 8AB $(V=9)$ would be separated $0.01 \mathrm{pc}\left(\right.$ or $\left.2.1 \times 10^{3} \mathrm{AU}\right)$ from HD 150136AB. Both the visual companion spectral type, derived from photometric considerations (later than $\mathrm{B} 3 \mathrm{~V}$ ), and the large distance from it to HD 150136AB conspire against the existence of a CWR between HD 150136AB and MIO 8AB. We will assume that, if existing, a CWR is located between HD 150136 A and B.

The stellar system is proposed as the ionizing source of the HII region RCW 108 (Yamaguchi et al. 1999).

ATCA 3 and $6 \mathrm{~cm}$ observations towards the system revealed one non-thermal source of about $2^{\prime \prime}$ size (at maximum angular resolution) superposed to HD 150136, and a thermal source on HD 150135. The flux densities measured were $S_{3 \mathrm{~cm}}=0.28 \pm 0.03 \mathrm{mJy}, S_{6 \mathrm{~cm}}=$ $0.1 \pm 0.04 \mathrm{mJy}$ for HD 150135, and $S_{3 \mathrm{~cm}}=2.61 \pm 0.03$ $\mathrm{mJy}$ and $S_{6 \mathrm{~cm}}=5.57 \pm 0.03 \mathrm{mJy}$ for HD 150136 .

The cluster NGC 6193 was observed with Chandra (Skinner et al. 2005), and the results are commented in Section 6 .

\section{ATCA Observations and Data Reduction}

Radio continuum observations of the four stars listed in Table 1 were obtained with the Australia Telescope Compact Array at 1.4 and 2.4 GHz. The star HD 93129A was also observed at 17.8 and $24.5 \mathrm{GHz}$.

\subsection{Observations at 1.4 and $2.4 \mathrm{GHz}$}

The observations towards the stars CD-47 4551 , HD 93129A, HD 124314, and HD 150136 were carried out in 2003 December, in the 6A configuration, simultaneously at $1.384 \mathrm{GHz}(20 \mathrm{~cm})$, and $2.368 \mathrm{GHz}$ $(13 \mathrm{~cm})$. The total bandwidth used at each frequency was $128 \mathrm{MHz}$. The average time on source was approximately $1 \mathrm{~h}$, except for HD 93129A, which was observed for $6 \mathrm{~h}$. The stars were observed during intervals of 10 to $15 \mathrm{~min}$, interleaved with short observations of closeby phase calibrators. The LST ranges spanned were $11 \mathrm{~h}$ for HD 93129A, $8 \mathrm{~h}$ for CD-47 4551 and HD 124314, and $6 \mathrm{~h}$ for HD 150136. The calibrator source PKS 1934-638 was used to set the flux density scale, with

Table 3. Measured flux densities

\begin{tabular}{lccccccc}
\hline Star & $t_{\text {int }}^{\mathrm{A}}[\mathrm{min}]$ & $S_{20 \mathrm{~cm}}[\mathrm{mJy}]$ & $S_{13 \mathrm{~cm}}[\mathrm{mJy}]$ & $S_{6 \mathrm{~cm}}[\mathrm{mJy}]$ & $S_{3 \mathrm{~cm}}[\mathrm{mJy}]$ & $\alpha_{13-20 \mathrm{~cm}}$ & $\alpha_{3-6 \mathrm{~cm}}$ \\
\hline $\mathrm{CD}-47^{\circ} 4551$ & 61 & $3.80 \pm 0.40$ & $3.82 \pm 0.35$ & $2.98 \pm 0.05$ & $1.77 \pm 0.05$ & $+0.01 \pm 0.02$ & $-0.89 \pm 0.06$ \\
HD 124314 & 71 & $2.82 \pm 0.40$ & $3.70 \pm 0.20$ & $4.14 \pm 0.2^{\mathrm{B}}$ & $2.78 \pm 0.1^{\mathrm{B}}$ & $+0.50 \pm 0.02$ & $-0.64 \pm 0.07$ \\
HD 150136 & 43 & $2.28 \pm 0.40$ & $3.05 \pm 0.55$ & $5.57 \pm 0.03$ & $2.61 \pm 0.03$ & $+0.54 \pm 0.02$ & $-1.29 \pm 0.03$ \\
\hline
\end{tabular}

${ }^{\text {A }}$ At 13/20 cm. ${ }^{\text {B }}$ Average of fluxes detected on 2000 March 30 and April 2. 
flux densities of $14.94 \mathrm{Jy}$ at $1.384 \mathrm{GHz}$ and $11.59 \mathrm{Jy}$ at $2.368 \mathrm{GHz}$.

The data were reduced and analyzed with the MIRIAD package. The calibrated visibilities were Fourier-transformed using 'robust' weighting, which provided the best signal-to-noise ratio combined with sidelobe suppression. The shortest baselines were excluded to avoid confusion of diffuse, extended emission.

\subsection{Observations at 17.8 and $24.5 \mathrm{GHz}$}

HD 93129A was recently found to be a wide binary formed by two O-type stars (Nelan et al. 2004). Its unique spectral type $\left(\mathrm{O} 2\right.$ If $\left.^{*}\right)$, and its radio detection at 4.8 and $8.64 \mathrm{GHz}$ as a non-thermal source (Benaglia \& Koribalski 2004) make it a very interesting stellar system. In order to get a radio spectrum from 1 to $25 \mathrm{GHz}, 12 \mathrm{~mm}$ observations were taken in 2004 May, at 17.8 and $24.5 \mathrm{GHz}(0.17$ and $0.12 \mathrm{~cm})$. Adverse weather conditions reduced the effective observing LST range time to around $4 \mathrm{~h}$. We alternately observed the frequency pairs of $17.728 / 17.856 \mathrm{GHz}$, and 24.428/24.556 GHz with $128 \mathrm{MHz}$ bandwidth each, to maximize the spectral range, sensitivity, and $U V$ coverage. The target scans were 6 min long, inserted between shorter phase calibrator scans. The absolute flux density was calibrated against Mars and PKS B0607-15.

\section{Emission Mechanisms}

The optically thick wind of a radio star emits thermal radiation by means of the bremsstrahlung mechanism (Wright \& Barlow 1975). At radio frequencies, the thermal flux density $\left(S_{v}^{\mathrm{T}}\right)$ is related to the stellar mass loss rate by

$$
\dot{M}\left[\mathrm{M}_{\odot} \mathrm{yr}^{-1}\right]=5.32 \times 10^{-4} \frac{\left(S_{v}^{\mathrm{T}}\right)^{3 / 4} d^{3 / 2} v_{\infty} \mu}{Z \sqrt{\psi g_{v} v}}
$$

Here $\psi$ is the mean number of electrons per ion, $Z$ is the rms ionic charge, and $g_{v}$ is the free-free Gaunt factor. In principle, the mass loss rate of an early-type star can be derived in a straightforward manner, by measuring the thermal radio flux density.

However, since the earliest observations of these stars, evidence of a spectral index reaching lower values than the thermal regime arose (Bieging, Abbott, \& Churchwell 1989). Emission characterized by such index is explained advocating the presence of energetic electrons embedded in the magnetic fields present in the winds that are involved in synchrotron emission. The total flux density will have a thermal $\left(S v^{\mathrm{T}} \propto v^{\alpha_{\mathrm{T}}}\right.$, with $\left.\alpha_{\mathrm{T}}=0.6-0.8\right)$ and a non-thermal $\left(S v^{\mathrm{NT}} \propto v^{\alpha_{\mathrm{NT}}}\right)$ contribution. It is widely accepted that the relativistic electrons are accelerated by the first-order Fermi mechanism in diffusive shocks (Bell 1978), and follow a power-law distribution of the form $N_{\mathrm{e}}(\gamma) \propto \gamma^{-p}$, where $p=2 \alpha_{\mathrm{NT}}+1$ and $\gamma$ is the electron Lorentz factor. The shocks can be present not only in single winds (White 1985) but, in the case of a binary system with interacting winds, at the CWR (Eichler \& Usov 1993).

As a result of the interaction between the copious UV photons, supplied by the $\mathrm{OB}$ star, and the relativistic electrons, high-energy emission by IC scattering (Chen \& White 1994) is also generated. The IC photon index $\Gamma\left(N_{\mathrm{ph}}(E) \propto E^{-\Gamma}\right)$ in the Thomson regime is related to $p$ by $p=2 \Gamma+1$ and hence with $\alpha_{\mathrm{NT}}$. If synchrotron and IC losses become important, the electron index is expected to change to $p^{\prime}=p+1$ (Longair 1997). The Lorentz factor at which the distribution changes $\left(\gamma_{b}\right)$ is a function of the average energy density of the photon field and the average time spent by the particles in the field region (Benaglia \& Romero 2003). This last variable depends, in turn, on the size of the non-thermal source and the velocity of the particles, $c / k$, where $k$ is a normalization parameter.

It is important to consider the effects capable of modifying the radio emission. Part of the synchrotron photons can be absorbed by the wind thermal ions (freefree absorption, FFA). The non-thermal flux can be expressed as $S_{v}^{\mathrm{NT}} \propto v^{\alpha_{\mathrm{NT}}} \exp \left(\tau_{0} v^{-2.1}\right)$, where $\tau_{0}$ is the optical depth at which the flux decreases in a factor of $1 / \mathrm{e}$ (Skinner et al. 1999). The thermal emission can also be absorbed by thermal ions in the so-called internal FFA. Synchrotron self-absorption (SSA) will affect the emission at low frequencies as $S(v) \propto v^{5 / 2}$. The critical frequency $v_{\mathrm{SSA}}$ where this mechanism acts can be expressed as (Williams 1963)

$$
v_{\mathrm{SSA}}[\mathrm{MHz}] \approx 2.145\left(\frac{S_{v_{\mathrm{SSA}}}}{\theta^{2}}\right)^{2 / 5} B^{1 / 5}
$$

where $\theta[\operatorname{arcsec}]$ is the angle subtended by the emitting region, $B[\mathrm{G}]$ is the magnetic field at the emitting region, and $S$ [mJy] is the non-thermal flux without absorption. The Razin-Tsytovitch effect (RTe) will be important for frequencies below $v_{\mathrm{RT}}=20 n_{\mathrm{e}} B^{-1} \mathrm{~Hz}$, where $n_{\mathrm{e}}$ is the electron density at the emitting region (Pachlolczyk 1970).

\section{Results}

All four stellar systems were detected at both 1.4 and 2.4 $\mathrm{GHz}$, while HD 93129A was also detected at 17.8 and $24.5 \mathrm{GHz}$ (see Figures 1-5). The detected radio sources are, as expected, unresolved. For CD-47 4551 , HD 124314, and HD 150136 the flux densities and spectral indices measured at 1.4 and $2.4 \mathrm{GHz}$ as well as previous measurements at 4.8 and $8.64 \mathrm{GHz}$ are given in Table 3 . The flux densities measured for HD 93129A from 1.4 to 24.5 GHz are given in Table 4. The quoted flux density uncertainties correspond to the measured $1 \sigma \mathrm{rms}$ in the corresponding ATCA image (see Figures 1-5).

The radio spectra based on the measured flux densities of all four stars show evidence for non-thermal emission suggesting that relativistic electrons are accelerated in shocks, like the ones present in wind-collision regions. The targets were selected because of their non-thermal emission at $3 / 6 \mathrm{~cm}$, and the trend is confirmed with the $13 / 20 \mathrm{~cm}$ data. The value for the HD 124314 fluxes at 3 and $6 \mathrm{~cm}$ (observed three times) were taken as the average of the fluxes detected closer in time, during the year 2000 (see Benaglia \& Koribalski 2004 for details). 
(a)

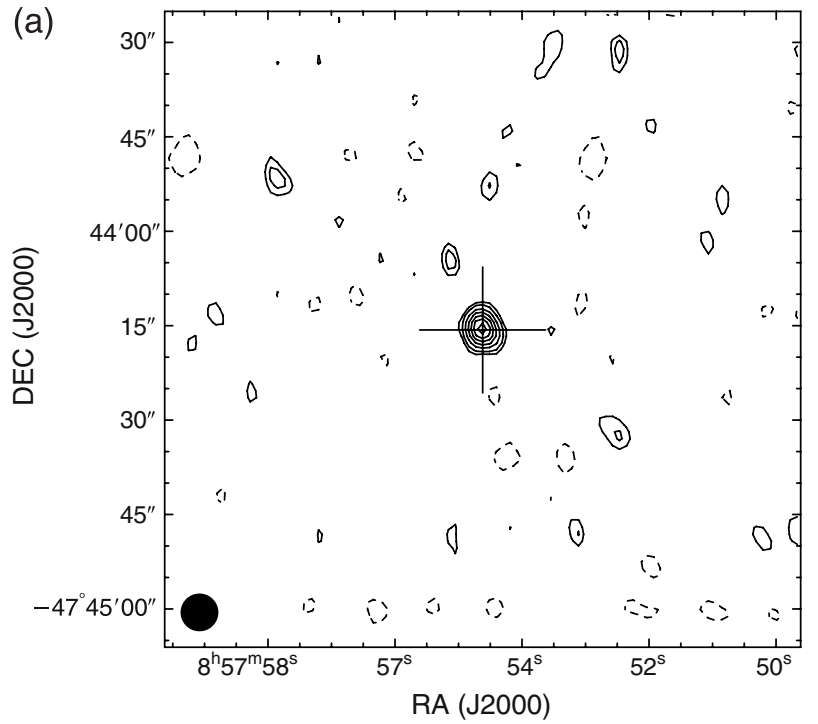

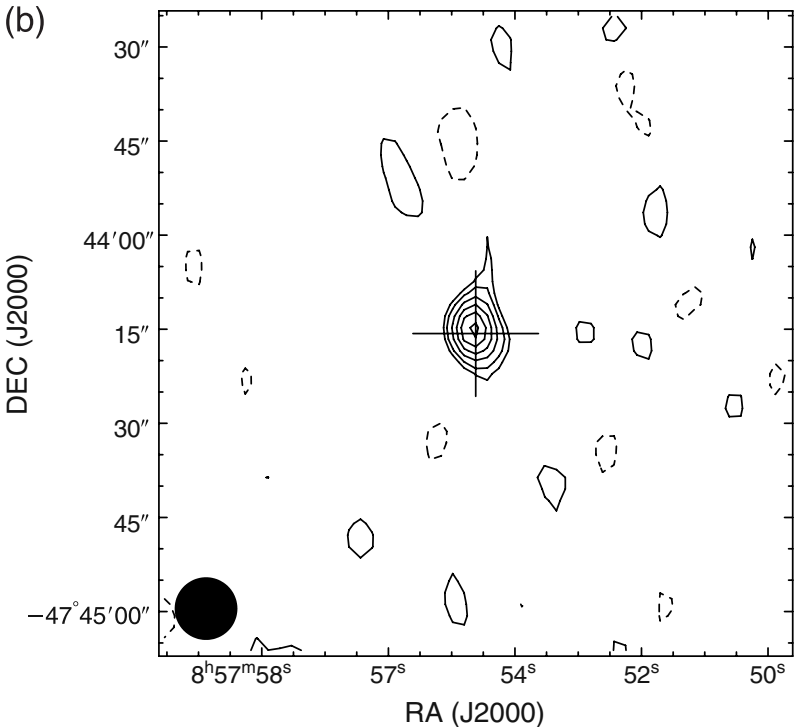

Figure 1 ATCA radio continuum images of CD-47 4551 at (a) $2.4 \mathrm{GHz}$ and (b) $1.4 \mathrm{GHz}$. In both images the optical position of the star is marked with a cross, and the synthesized beam $\left(6^{\prime \prime}\right.$ at $2.4 \mathrm{GHz}$, and $10^{\prime \prime}$ at $\left.1.4 \mathrm{GHz}\right)$ is displayed in the bottom left corner. The contour levels are (a) $-0.7,0.7(2 \sigma), 1.0,1.5,2.0,2.5,3.0$, and $3.5 \mathrm{mJy}^{\text {beam }}{ }^{-1}$ and $(\mathrm{b})-0.8,0.8(2 \sigma), 1.2,1.8,2.4,3.0$, and $3.6 \mathrm{mJy}^{\mathrm{beam}}{ }^{-1}$.

(a)

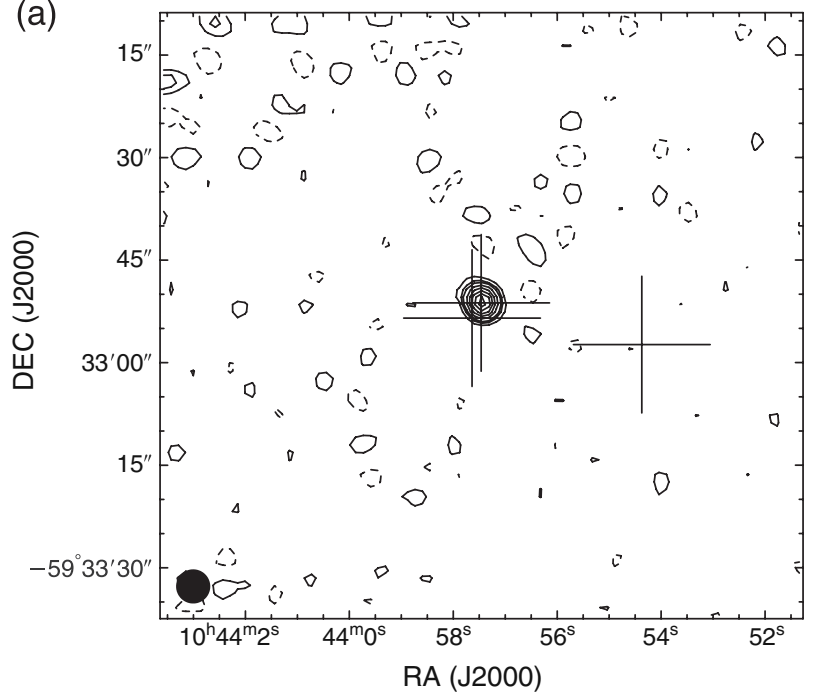

(b)

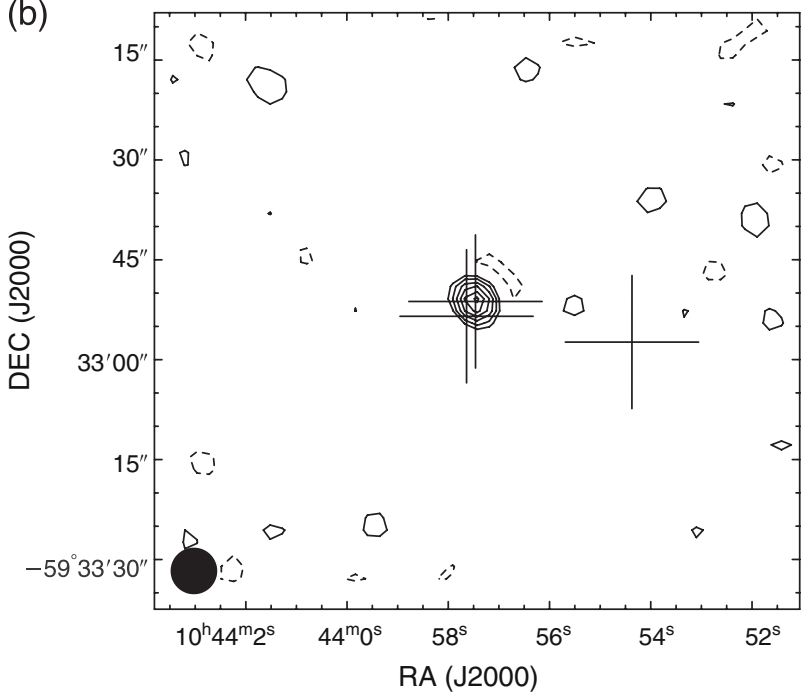

Figure 2 ATCA radio continuum images of $\mathrm{HD} 93129 \mathrm{~A}$ at (a) $2.4 \mathrm{GHz}$ and (b) $1.4 \mathrm{GHz}$. In each image the optical positions of the stars HD 93129B, HD 93129A, and HD 93128 (from left to right) are marked with a cross. The synthesized beam ( $5^{\prime \prime}$ at $2.4 \mathrm{GHz}$, and $7^{\prime \prime}$ at $1.4 \mathrm{GHz}$ ) is displayed in the bottom left corner. The contour levels are (a) $-0.8,0.8(2 \sigma), 1.5,2,3, \ldots$, and $7 \mathrm{mJy} \mathrm{beam}^{-1}$ and (b) $-1.8,1.8(2 \sigma), 3.0,4.5,6.0$, 7.5 , and $9.0 \mathrm{mJy}^{\text {beam }}{ }^{-1}$.

Table 4. Flux densities for HD 93129A

\begin{tabular}{lcccc}
\hline Obs. date & $\begin{array}{c}t_{\text {int }} \\
{[\mathrm{min}]}\end{array}$ & $\begin{array}{c}\lambda \\
{[\mathrm{cm}]}\end{array}$ & $\begin{array}{c}v \\
{[\mathrm{GHz}]}\end{array}$ & $\begin{array}{c}S_{\lambda} \\
{[\mathrm{mJy}]}\end{array}$ \\
\hline 2003 December 20 & 362 & 20 & 1.4 & $9.4 \pm 0.9$ \\
2003 December 20 & 362 & 13 & 2.4 & $7.8 \pm 0.4$ \\
2003 January 28 & 298 & 6 & 4.8 & $4.1 \pm 0.4$ \\
2003 January 28 & 298 & 3 & 8.64 & $2.0 \pm 0.2$ \\
2004 May 5 & 52 & 0.17 & 17.8 & $1.8 \pm 0.15$ \\
2004 May 5 & 48 & 0.12 & 24.5 & $1.5 \pm 0.35$ \\
\hline
\end{tabular}

While imaging HD 93129A at 17.8 and $24.5 \mathrm{GHz}$ we discovered a new radio source (see Figure 6). Its position is $(\mathrm{RA}, \text { Dec })_{\mathrm{J} 2000}=\left(10^{\mathrm{h}} 43^{\mathrm{m}} 59.99^{\mathrm{s}},-59^{\circ} 33^{\prime} 09.2^{\prime \prime}\right)$, very close to the star DETWC Tr 14 J104359.9-593309 (DeGioiaEastwood et al. 2001). The measured flux densities are $S_{17.8 \mathrm{GHz}}=2.2 \pm 0.2 \mathrm{mJy}$ and $S_{24.5 \mathrm{GHz}}=2.6 \pm 0.5 \mathrm{mJy}$. The corresponding spectral index is $0.6 \pm 0.3$, namely as expected for thermal bremsstrahlung emission from a stellar wind. The visual magnitudes for this star are $V=13.46$ and $B=14.76$. If it belongs to the $\operatorname{Tr} 14$ cluster $(d \approx 2.5 \mathrm{kpc})$, the spectral type should be between $\mathrm{B} 0 \mathrm{~V}$ and $\mathrm{B} 2 \mathrm{~V}$. 
(a)

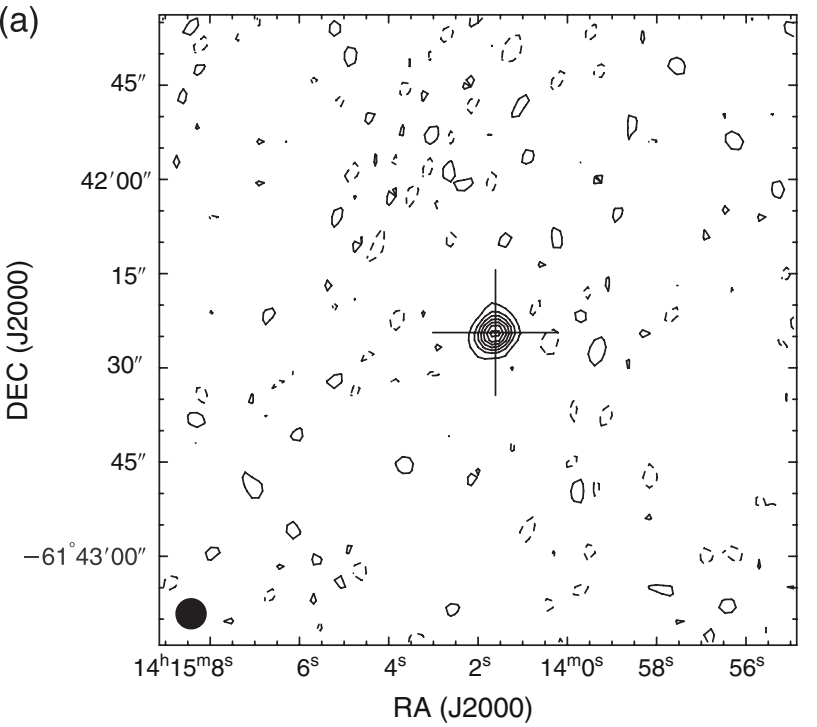

(b)

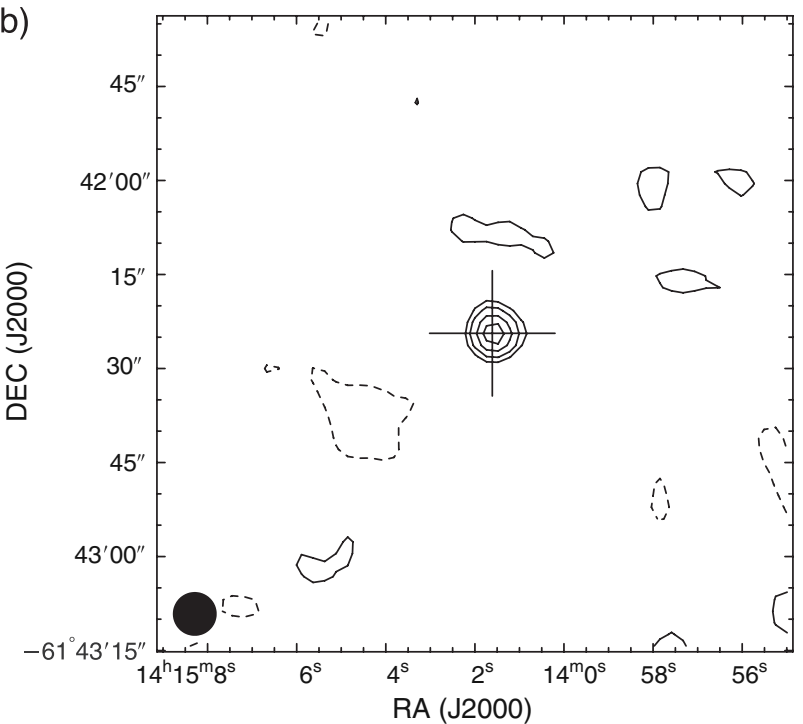

Figure 3 ATCA radio continuum images of HD 124314 at (a) $2.4 \mathrm{GHz}$ and (b) $1.4 \mathrm{GHz}$. In both images the optical position of the star is marked with a cross, and the synthesized beam $\left(5^{\prime \prime}\right.$ at $2.4 \mathrm{GHz}$, and $7^{\prime \prime}$ at $\left.1.4 \mathrm{GHz}\right)$ is displayed in the bottom left corner. The contour levels are (a) $-0.4,0.4(2 \sigma), 1.0,1.5,2.0,2.5,3.0$, and $3.5 \mathrm{mJy}^{-1}$ beam $^{-1}$ and (b) $-0.8,0.8(2 \sigma), 1.2,1.6,2.0$, and $2.4 \mathrm{mJy} \mathrm{beam}^{-1}$.
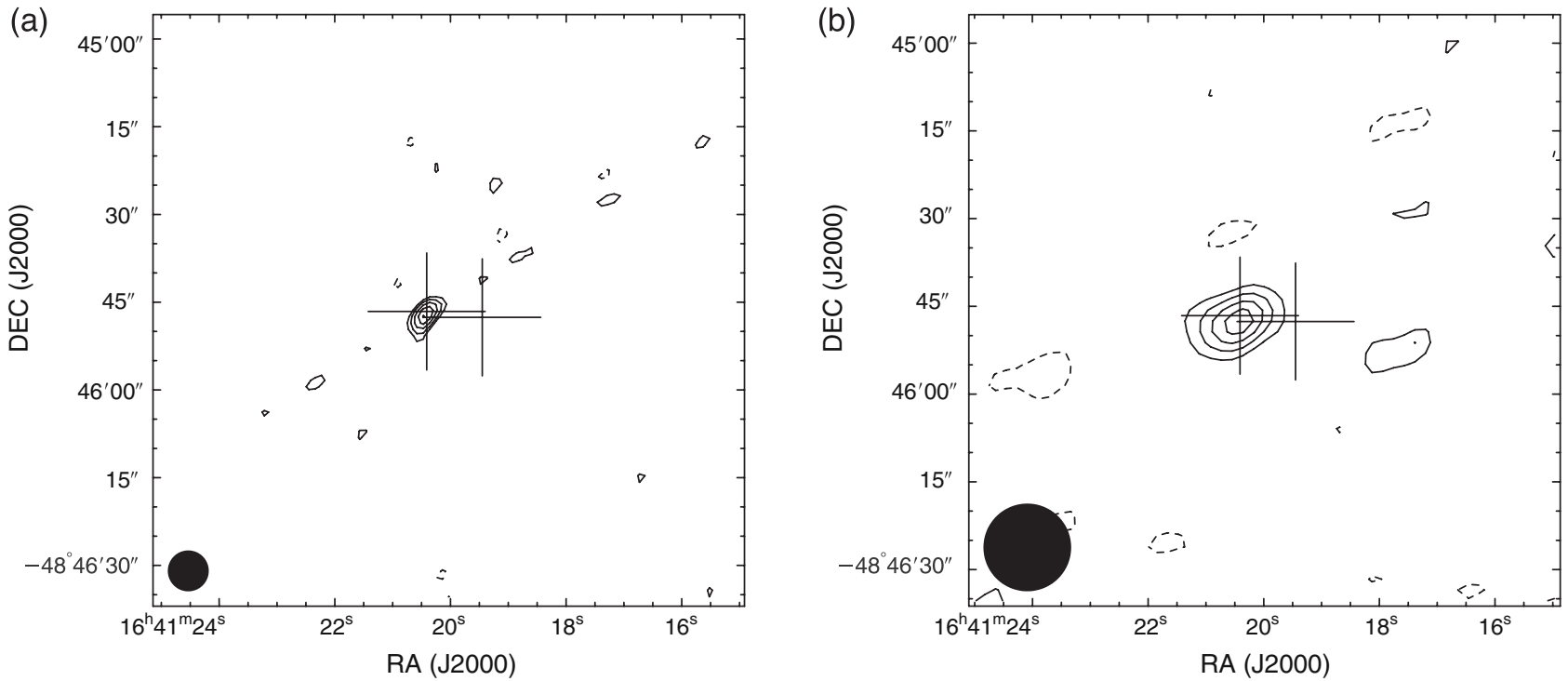

Figure 4 ATCA radio continuum images of HD 150136 at (a) $2.4 \mathrm{GHz}$ and (b) $1.4 \mathrm{GHz}$. In each image the optical positions of the stars HD 150136 (left) and HD 150135 (right), are marked with a cross. The synthesized beam $\left(7^{\prime \prime}\right.$ at $2.4 \mathrm{GHz}$, and $15^{\prime \prime}$ at $1.4 \mathrm{GHz}$ ) is displayed in the bottom left corner. The contour levels are (a) $-1.1,1.1(2 \sigma), 1.5,2.0,2.5$, and $3.0 \mathrm{mJy}^{-1}$ beam $^{-1}$ and (b) $-0.8,0.8(2 \sigma), 1.2,1.6$, and $2.0 \mathrm{mJy}^{\text {beam }}{ }^{-1}$ (right).

\subsection{Non-Thermal Emission}

The measured fluxes of CD-47 4551 , HD 124314, and HD 150136 presented turnovers along the frequency axis (see Tables 3 and 4). The flux of HD 93129A diminished with frequencies, but the slighter decrease between 1.4 and $2.4 \mathrm{GHz}$ is indicative that the turnover is beginning to appear towards shorter frequencies. Processes like SSA, RTe, and FFA can contribute to the turnover. Unfortunately, due to the lack of data below $1.4 \mathrm{GHz}$, and the fact that the turnover is poorly defined, it was not possible to determine which process - and in which manner - shape the spectra at low frequencies.
The strong non-thermal emission found at the stellar spectra is not easy to isolate and characterize, as the processes mentioned above affect it severely. The emitting regions were detected as ATCA point sources, yielding no information on source sizes; additionally the observational dataset for each star, although covering an important frequency interval, is reduced to four points (six in the case of HD 93129A). Moreover, the flux from a CWR varies with time, depending on the orbit and the relative positions of the two components in a binary system.

However, a rough estimation of the non-thermal spectral index can be done under the following simplifying assumptions. 
(a)

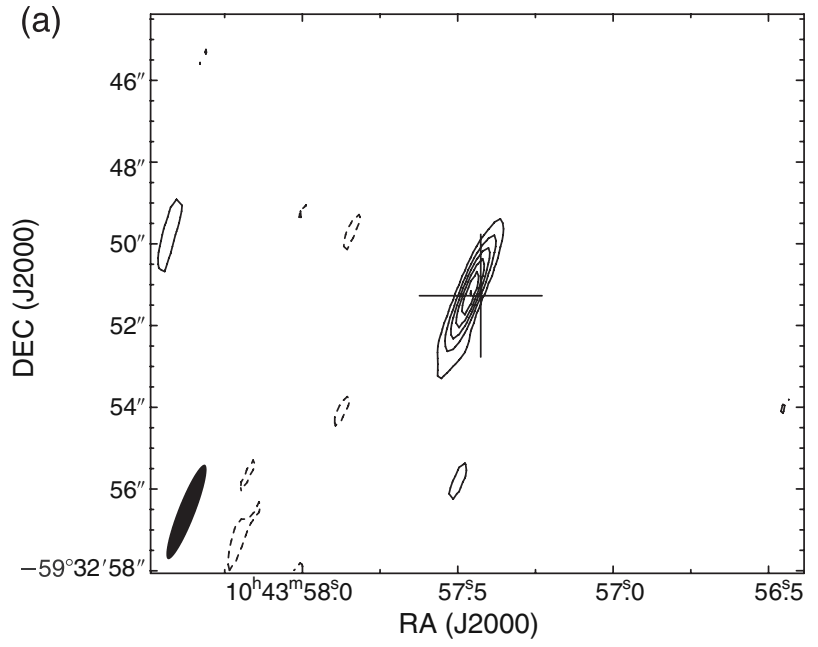

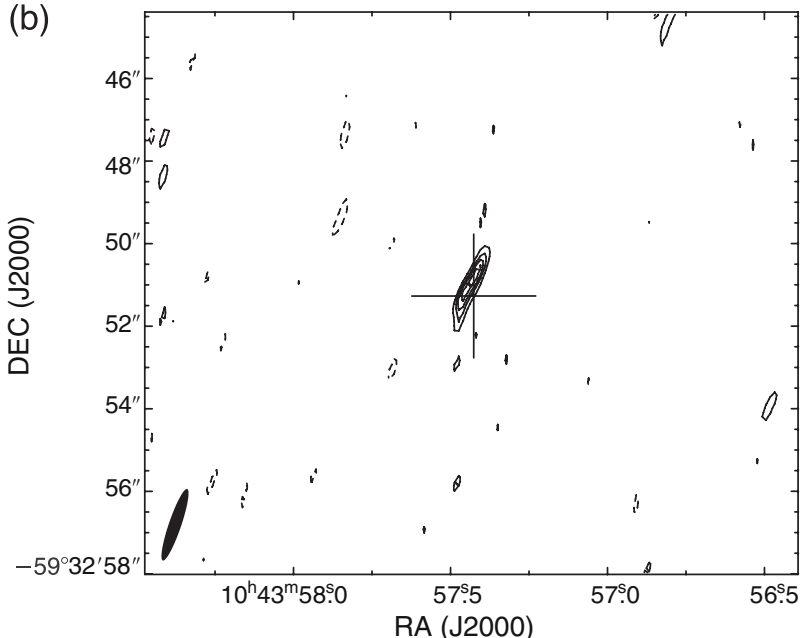

Figure 5 ATCA radio continuum images of HD 93129A at (a) $17.8 \mathrm{GHz}$ and (b) $24.5 \mathrm{GHz}$. In both images the optical position of the star is marked with a cross, and the synthesized beam $\left(2.4^{\prime \prime} \times 0.4^{\prime \prime}\right.$ at $17.8 \mathrm{GHz}$, and $1.8^{\prime \prime} \times 0.3^{\prime \prime}$ at $\left.24.5 \mathrm{GHz}\right)$ is displayed in the bottom left corner. The contour levels are (a) $-0.3,0.3(2 \sigma), 0.6,0.9,1.2,1.5$, and $1.8 \mathrm{mJy}_{\text {beam }}{ }^{-1}$ and (b) $-0.7,0.7(2 \sigma), 1.0,1.2$, and $1.4 \mathrm{mJy} \mathrm{beam}^{-1}$.

(a)

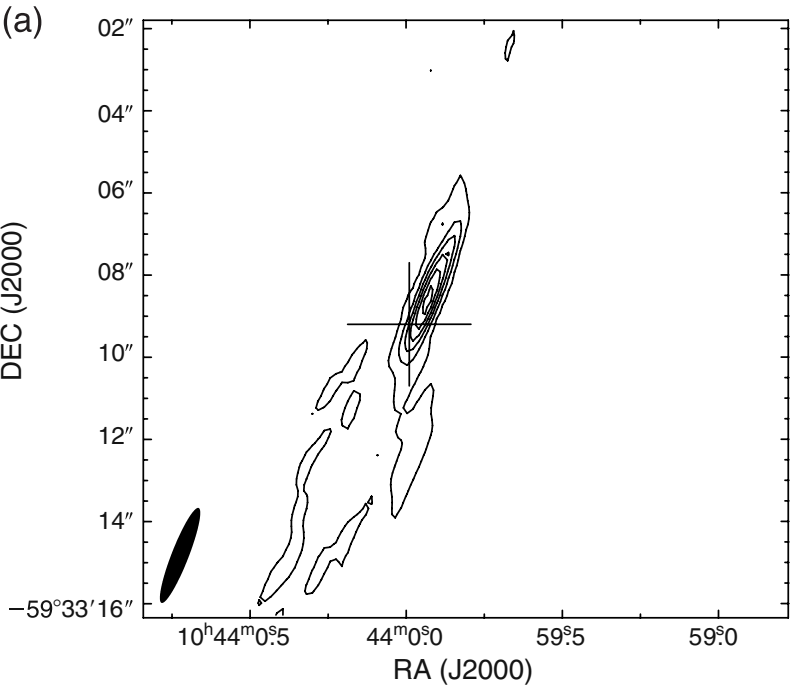

(b)

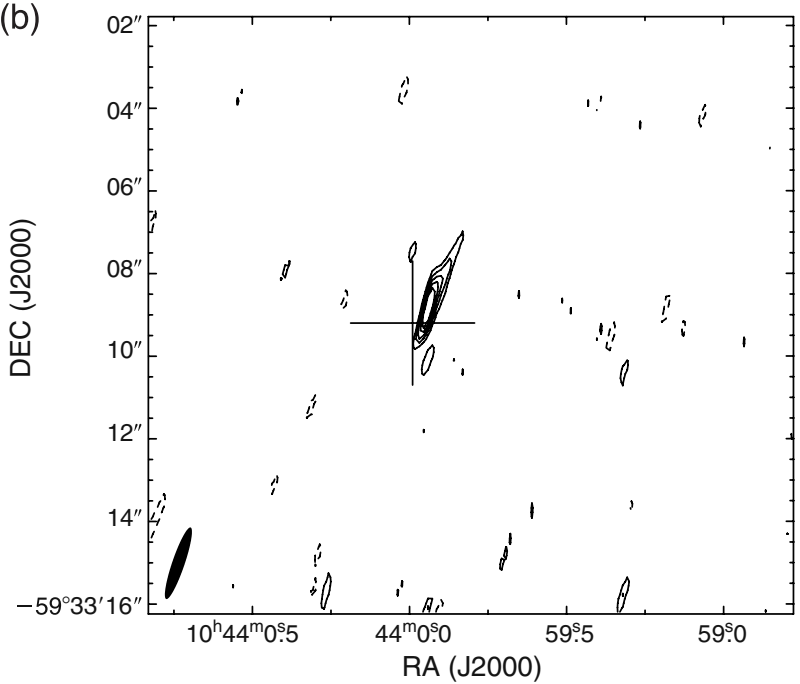

Figure 6 ATCA radio continuum images of the star DETWC Tr 14 J104359.9-593309 (DeGioia-Eastwood et al. 2001) at (a) 17.8 GHz and (b) $24.5 \mathrm{GHz}$. In both images the optical position of the star is marked with a cross, and the synthesized beam $\left(2.4^{\prime \prime} \times 0.4^{\prime \prime}\right.$ at $17.8 \mathrm{GHz}$, and $1.8^{\prime \prime} \times$ $0.3^{\prime \prime}$ at $24.5 \mathrm{GHz}$ ) is displayed in the bottom left corner. The contour levels are (a) $-0.4,0.4(2 \sigma), 0.8,1.2,1.6,2$, and $2.4 \mathrm{mJy}^{-1}$ beam $^{-1}$ and (b) $-1,1$ $(2 \sigma), 1.4,1.8,2.2$, and $2.6 \mathrm{mJy}_{\text {beam }}{ }^{-1}$.

We have analyzed the simultaneity of the radio measurements. In the case of HD 93129A, on a first approximation, all the observations can be considered simultaneously taken: The interval between observations was 18 months; the interpretation of very new HST ACS/HRC data combined with the former FGS observations is consistent with a binary of period $\approx 50 \mathrm{yr}$ (Maíz-Apellániz et al. 2006). The observations of CD-47 4551 and HD 124314 covered a time interval of $\approx 3.5 \mathrm{yr}$. Optical monitoring of radial velocities is needed to test the condition for CD- $47^{\circ} 4551$ and HD 124314. On the contrary, the work on HD 150136 by Niemela \& Gamen (2005) established that the (O3.5 I+ O6 V) form a close pair (period 2.7 days). That means that each of the radio flux densities presented here should be taken as a mean value, averaged over LST ranges of several hours, and precludes any non-thermal flux estimate. Thus, we have assumed that the radio flux densities for CD- $47^{\circ}$ 4551, HD 93129A, and HD 124314 are quasi-simultaneous, despite the hours interval elapsed during observation.

The search for early-type companions of OB stars has been progressing successfully (Niemela et al. 2006; Rauw et al. 2005), making the existence of single stars with detectable synchrotron radiation look doubtful. Although the binary nature of CD- $47^{\circ} 4551$ has to be studied, and the 'SB1?' tag of HD 124314 has yet to be confirmed, it seems reasonable to assume that the stars are both $(\mathrm{O}+\mathrm{OB})$ binaries and the non-thermal radiation can be produced at a CWR in each system.

If the thermal contribution $S^{\mathrm{T}}$ can be subtracted from the observed flux densities $(S)$ at frequencies large enough for 
Table 5. Estimates of NT indices at $3-6 \mathrm{~cm}$

\begin{tabular}{lccc}
\hline Star & $S_{3 \mathrm{~cm}}^{\mathrm{T}}[\mathrm{mJy}]$ & $S_{3 \mathrm{~cm}}^{\mathrm{NT}}[\mathrm{mJy}]$ & $\alpha_{3-6 \mathrm{~cm}}^{\mathrm{NT}}$ \\
\hline CD-47 4551 & 0.16 & 1.61 & $\sim-1.0$ \\
HD 93129A & 0.50 & 1.50 & $\sim-1.5$ \\
HD 124314 & 0.04 & 2.74 & $\sim-0.7$ \\
\hline
\end{tabular}

which the effects of absorption mechanisms minimize, an un-absorbed non-thermal flux will be obtained. We proceeded to compute $\alpha^{\mathrm{NT}}$ between fluxes at 3 and $6 \mathrm{~cm}$, in the way explained below.

We assumed the mass loss rate of each star/system as listed in Table 1, and computed the values for $S^{\mathrm{T}}$ from Eqn (1) at $3 \mathrm{~cm}(8.64 \mathrm{GHz})$ for $\gamma=Z=1$. The flux values at $6 / 3 \mathrm{~cm}$ showed also, in general, lower relative errors than the rest, and they were taken simultaneously. For an average thermal spectral index of +0.7 , the thermal flux at $6 \mathrm{~cm}$ could be derived. With the total and the thermal fluxes at 3 and $6 \mathrm{~cm}$, the estimates of the respective non-thermal flux and the corresponding spectral index were performed. The resulting values are given in Table 5 .

Figure 7 displays, besides the ATCA fluxes, the subtracted thermal contribution and the residual flux at each observed frequency. It can be appreciated that the values obtained for the thermal contribution in each case were always much smaller that the non-thermal ones: The non-thermal CWR emission seems to dominate over any thermal emission from the individual stellar winds.

\section{Stellar X-ray Emission}

OB stars - either single or multiple — are known as X-ray detectable emitters (Berghöfer et al. 1997; Chlebowski \& Garmany 1991), with X-ray luminosities $>>10^{-7} L_{\text {bol }}$. However, some X-ray features are considered as evidence of a CWR, like larger luminosities, or the presence of the $\mathrm{Fe}_{\mathrm{K} \alpha}$ emission line, produced at widebinaries collisions events.

Whereas low-mass flare activity produces a highly correlated behaviour between X-ray and radio emission, for $\mathrm{OB}$ stars in contrast such emission mechanisms remain open to different interpretations.

The four target stars have been detected as non-thermal radio emitters. They are very hot, luminous, and massive. One is cataloged as a binary with another early-type star (HD 93129A) and it was detected at radio waves despite its large distance $(2.5 \mathrm{kpc})$. HD 124314 is also a binary, and presented flux variations up to $40 \%$ (Benaglia et al. 2001). HD 150136 belongs to a multiple system with other OB stars. Finally, there is no binary information about the fourth object, CD $-47^{\circ} 4551$. We proceeded to investigate their $\mathrm{X}$-ray emission from literature data, in order to check if they fulfill the $L_{\mathrm{X}} / L_{\text {bol }}$ ratio for OB stars, if in any case this ratio exceeds the canonical value (indicative of strong shocks at a CWR), and, if spectroscopic data were available, to look for the $\mathrm{Fe}_{\mathrm{K} \alpha}$ complex.
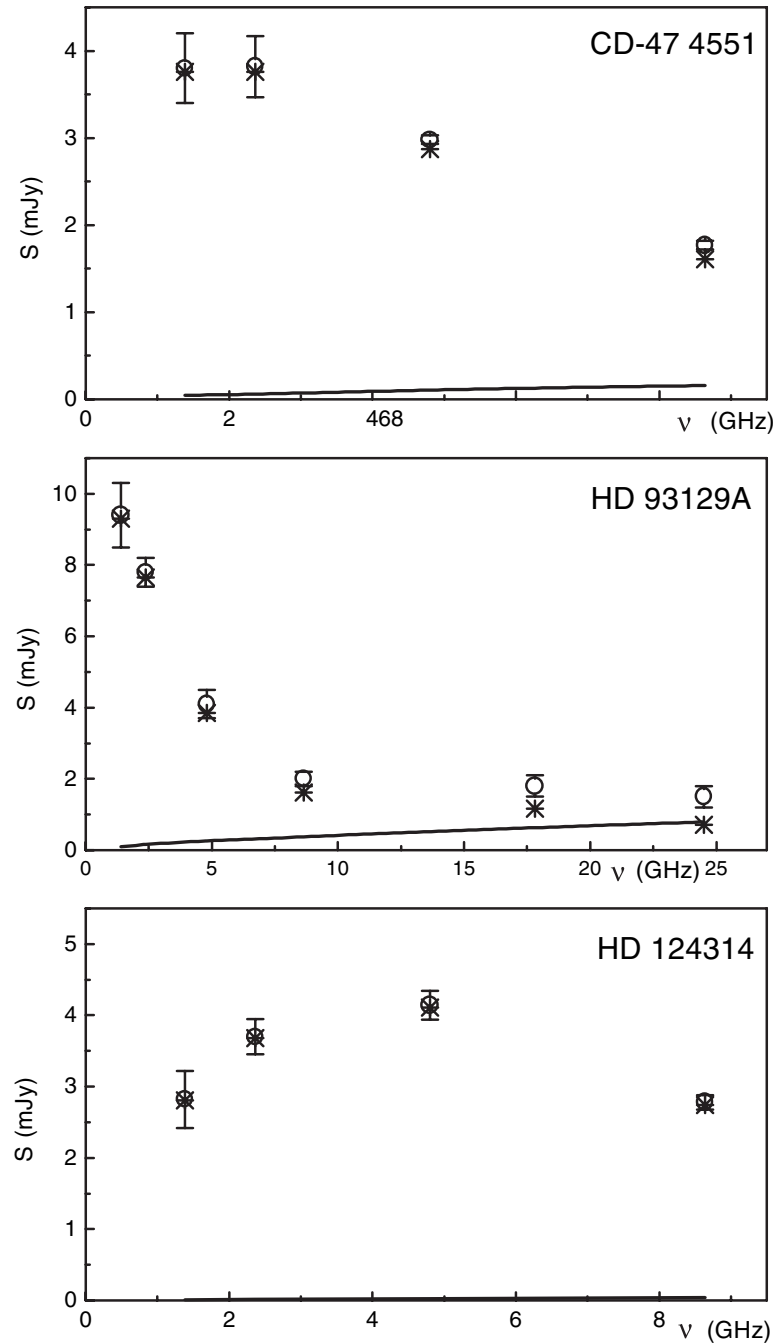

Figure 7 Radio flux densities of the stars CD- $47^{\circ} 4551$, HD 93129A, and HD 124314. The open circles mark our measurements, the line indicates the fit for the thermal contribution, and the asterisks, the residuals.

\section{$6.1 X$-rays from $C D-47^{\circ} 4551$}

ROSAT archive data were found on the field of CD- $47^{\circ}$ 4551. The investigation on stellar X-rays was performed using 4 ROSAT-PSPC X-ray observations towards this star (see Table 6). The angular resolution of these data is about $45 \mathrm{arcsec}$. The image FOV is $6.32^{\circ} \times 6.32^{\circ}$ and the scale is $15^{\prime \prime}$ per pixel. Our analysis was carried out using the XIMAGE and PIMMS software.

We first extracted the photon count rates over a radius of $2^{\prime}$, which include more that $90 \%$ of the PSF encircled energy. Due to the high pixel scale, the circle encompasses other optical (Digitized Sky Survey: DSS ${ }^{3}$ ) sources in the field (see Figure 8a), though the resolution of the X-ray data does not allow to distinguish between emission from nearby stars. However, only two B stars were cataloged over that circle according to the SIMBAD database, enabling us to

${ }^{3}$ The Digitized Sky Survey was produced at the Space Telescope Science Institute under the US Government grant NAG W-2166. 
Table 6. ROSAT X-ray observations of CD- $47^{\circ} 4551$

\begin{tabular}{llccc}
\hline ROSAT data & \multicolumn{1}{c}{ Start obs. date } & Exposure time [s] & Offset $^{\mathrm{A}}\left[{ }^{\circ}\right]$ & Id. no. \\
\hline rs932518n00 & 1990 October 30 & 25642 & 3.54 & 1 \\
rs932519n00 & 1990 November 11 & 22941 & 4.04 & 2 \\
rs932616n00 & 1990 October 30 & 29050 & 4.15 & 3 \\
rs932617n00 & 1990 June 11 & 21783 & 3.63 & 4 \\
\hline
\end{tabular}

${ }^{A}$ Refers to the angular distance between the FOV centre and the position of CD- $47^{\circ} 4551$.
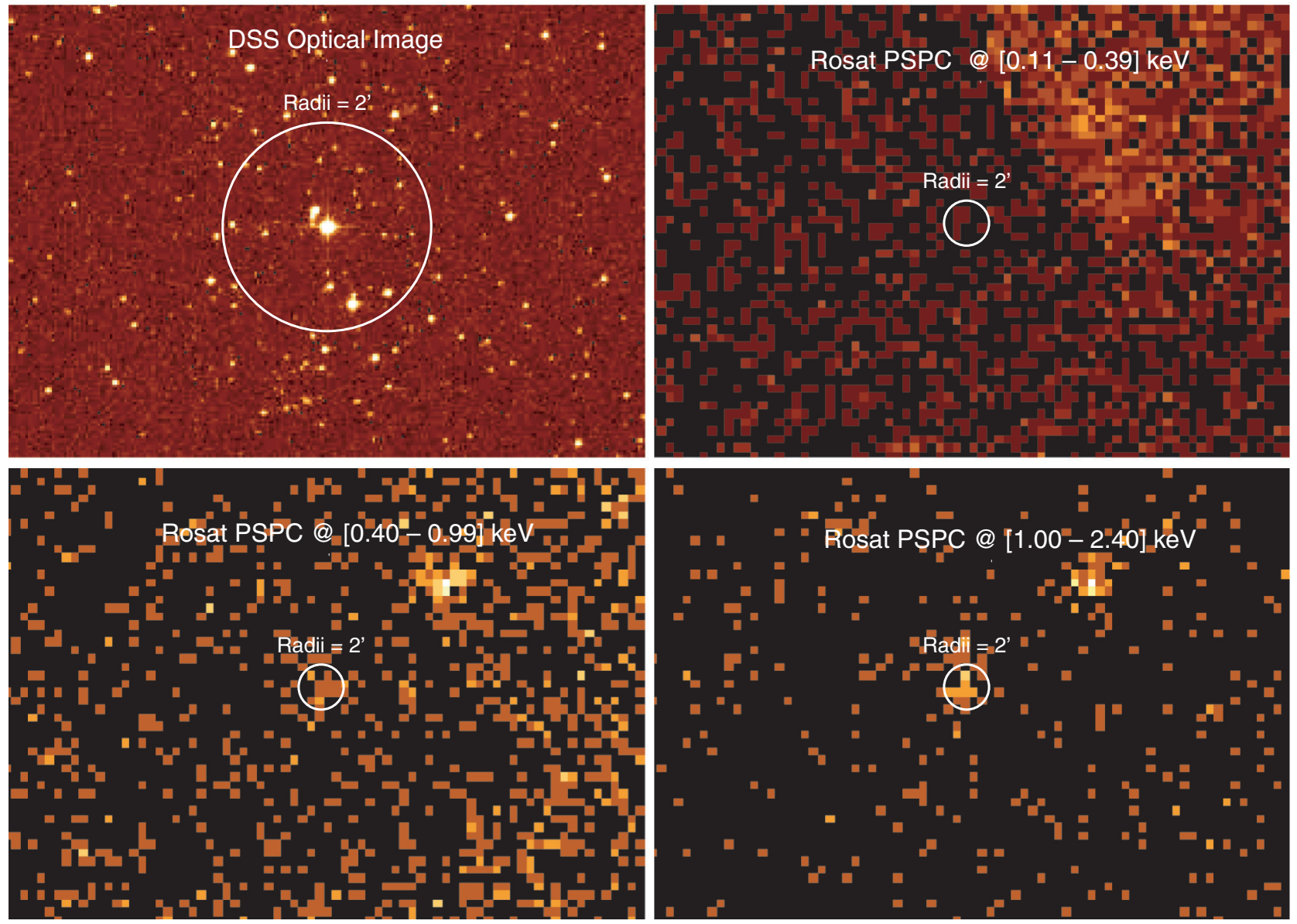

Figure 8 Optical and X-ray observation of the CD- $47^{\circ} 4551$ star. (a) DSS field towards CD- $47^{\circ} 4551$. (b-d) ROSAT emission at the bands (b) $0.11-0.39$, (c) $0.40-0.99$, and (d) $1.0-2.4 \mathrm{keV}$.

disregard their X-ray contribution, and conclude that the O4 III star dominates the observed X-ray emission.

According to the different ROSAT energy images (Figure 8b-8d), the observed X-ray emission appears quite hard, above $1 \mathrm{keV}$. We measured count rates in three $\mathrm{X}$-ray bands - a wide band at $0.1-2.4 \mathrm{keV}$, a soft band (S) at $0.1-0.5 \mathrm{keV}$, and a hard band $(\mathrm{H})$ between $0.5-2.0 \mathrm{keV}$. Furthermore, we estimated the hardness ratio, $(\mathrm{H}-\mathrm{S}) /(\mathrm{H}+\mathrm{S})$, yielding an average of $0.91 \pm 0.09$. This result implies that most (about 70\%) of the emitted X-ray energy lies between 1.0 and $2.4 \mathrm{keV}$ (see Table 7). The low signal-to-noise ratio of the observation prevents us from estimating count-rate error values, although we confirm that the average total count rate for $\mathrm{CD}-47^{\circ} 4551$ is $1.13 \pm 0.08 \times 10^{-3} \mathrm{cts} \mathrm{s}^{-1}$.
It was previously settled that non-thermal radio emission is produced by the synchrotron mechanism, and can be described at $3 / 6 \mathrm{~cm}$ by an index $\alpha_{\mathrm{NT}}=-1.0$ (see Section 5). The X-ray emission is hard; the scattering processes are more important at several stellar radii where the UV photon density is larger. It will be expected then that inverse Compton processes are responsible for the observed hard $\mathrm{X}$-ray emission, as well as that absorption processes are related to the outer layers of the stellar wind. We have assumed that $\mathrm{X}$-rays originate in wind layers external enough to be almost un-affected by stellar absorption, enabling us to neglect the wind-X-ray interaction. A strong shock in the $\mathrm{O} 4 \mathrm{III}$ star is originated when the wind reaches velocities close to the terminal velocity, at several 
Table 7. Source count rates $\left[10^{-3} \mathrm{cnt} \mathrm{s}^{-1}\right]$ and hardness ratio for CD-47 4551

\begin{tabular}{lcccc}
\hline Id. no. & Total $(0.1-2.4)$ & Soft $(0.1-0.5)$ & Hard $(0.5-2.4)$ & Hardness ratio $(\mathrm{H}-\mathrm{S}) /(\mathrm{H}+\mathrm{S})$ \\
\hline 1 & 1.13 & 0.04 & 1.05 & 0.92 \\
2 & 1.23 & 0.03 & 1.13 & 0.94 \\
3 & 0.89 & 0.07 & 0.79 & 0.83 \\
4 & 1.28 & 0.05 & 1.15 & 0.91 \\
Average & $1.13 \pm 0.08$ & $0.05 \pm 0.02$ & $1.08 \pm 0.08$ & $0.91 \pm 0.09$ \\
\hline
\end{tabular}

Table 8. XMM-Newton X-ray observations of HD 93129A

\begin{tabular}{|c|c|c|c|c|c|c|}
\hline \multirow[t]{2}{*}{ Obs id. } & \multirow[t]{2}{*}{$X M M-N e w t o n$ data set } & \multirow[t]{2}{*}{ Start obs. date } & \multirow[t]{2}{*}{ Exposure time $^{\mathrm{A}}[\mathrm{s}]$} & \multicolumn{3}{|c|}{ Count rate $\left[10^{-3} \mathrm{cnt} \mathrm{s}^{-1}\right]$} \\
\hline & & & & PN & MOS1 & MOS2 \\
\hline 1 & 0112560101 & 2001 June 25 & 14000 & - & 53.8 & 47.5 \\
\hline 2 & 0112560201 & 2001 June 28 & 29000 & 151.1 & 53.1 & 45.0 \\
\hline 3 & 0112560301 & 2001 June 30 & 31000 & 148.2 & 50.6 & 48.9 \\
\hline
\end{tabular}

A The values are quite approximate because of the slight difference between exposure time of the MOS1, MOS2, and PN observations.

stellar radii. At this point, the wind density (and thus the absorption produced by the wind) is very low. We considered that only neutral absorption processes associated with the ISM are affecting the X-rays from this source, and adopt a cold absorption model.

We calculated the total Galactic Hi column density using the Web version of the $N_{\mathrm{HI}_{\mathrm{I}}}$-FTOOL software ${ }^{4}$, and estimated an interstellar column of neutral hydrogen $N_{\mathrm{HI}}$ of $0.92 \times 10^{22} \mathrm{~cm}^{-2}$.

The X-ray count rate can be converted to fluxes using PIMMS, yielding an un-absorbed flux of $1.35 \times 10^{-13}$, $0.024 \times 10^{-13}$, and $0.76 \times 10^{-13} \mathrm{erg} \mathrm{cm}^{-2} \mathrm{~s}^{-1}$ for the total, soft, and hard bands, respectively.

At a distance of $1.3 \mathrm{kpc}$, the total X-ray luminosity of $\mathrm{CD}-47^{\circ} 4551$ is then $L_{\mathrm{X}} \sim 2.73 \times 10^{31} \mathrm{erg} \mathrm{s}^{-1}$. From the calibration tables of Martins et al. (2005), we adopt a value $\log \left(L_{\mathrm{bol}} / \mathrm{L}_{\odot}\right)=5.85$, and $L_{\mathrm{X}} / L_{\mathrm{bol}}$ is $1.2 \times 10^{-8}$.

\section{$6.2 X$-rays from HD 93129A}

The target HD 93129A is placed at the core of the Trumpler 14 open cluster in the Carina Nebula, one of the most observed regions in X-rays. We found ten available $X M M$-Newton X-ray observations towards this particular stellar system. Seven of these were centered at the LBV $\eta$ Carinae star, while the rest are centered at the WR 25 system (WN7+abs). At these pointing directions HD 93129A shows an offset of $11.767^{\prime \prime}$ and $10.455^{\prime \prime}$, respectively. These differences imply changes in the PSF as well as the encircled energy radii: in the most favourable situation (observations centered at WR 25, see Table 8), the $90 \%$ of encircled energy needs an extraction radius of $40^{\prime \prime}$. In order to disentangle the contamination from nearby sources in the resulting HD 93129A spectrum, we used an

\footnotetext{
${ }^{4}$ heasarc.gsfc.nasa.gov/cgi-bin/Tools/w3nh/w3nh.pl
}

extraction radius of $11^{\prime \prime}$, in which the fraction of the encircled energy is only about $60 \%$. In doing this, we favoured the extraction of an uncontaminated HD93129A spectrum against losing some X-ray photons from the external zone of the PSF (downgrading statistics). The final angular resolution of the image resulted in $\approx 6$ arcsec, over a FOV of 30 arcmin.

Most massive stars are known to display thermal X-ray spectra with strong and broad emission lines (Kahn et al. 2001; Cohen et al. 2003), contributed by non-thermal emission arising from IC scattering of UV photons due to the presence of a population of relativistic electrons (Pollock 1987; Chen \& White 1991; Eichler \& Usov 1993). However, HD 93129A seems to display several emission lines from low to high energies (see Figure 9). Such emission lines can only be produced by high-temperature thermal plasma; consequently, the spectral analysis presented here was performed using solely a combination of thin thermal emission models.

Our spectral fits were performed using combined data from all EPIC cameras, in order to improve statistics and discard any instrumental dependence that could be produced by the different responses of the detectors. The spectral model fitted consisted of a combination of absorption and emission models. Two separate absorption columns were used: (a) one based on the WABS atomic cross section (Morrison \& McCammon 1983) to account for the action of the circumstellar medium, and (b) one with the ionized absorber model ABSORI (Done et al. 1992) to include the effect of the stellar wind absorption processes. The first absorption component is due to the neutral material and hydrogen column density, which was calculated and fixed at $N_{\mathrm{H}}=0.52 \times E(B-V) \times 10^{22} \mathrm{~cm}^{-2}=0.286 \times 10^{22} \mathrm{~cm}^{-2}$ (Shull \& van Steemberg 1985). Here $(B-V)=0.22$ (Walborn et al. 2002). Table 9 gives the results of the fits. 


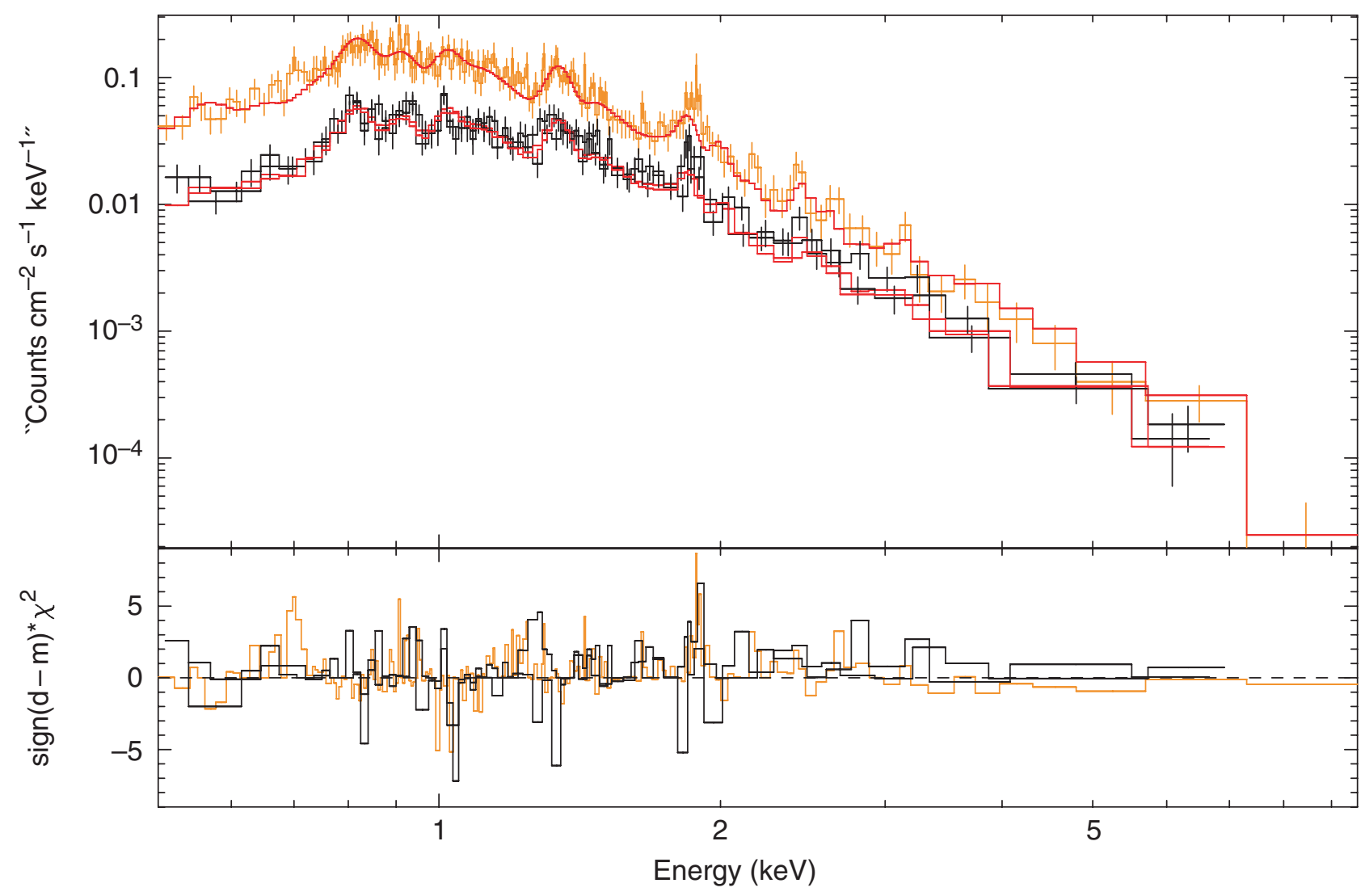

Figure 9 EPIC-PN X-ray spectra of HD 93129A. The PN and MOS spectra were binned to reach a minimum of four and nine counts per channel, respectively. The red line shows the best fit model. The broad observed emission lines (line blending effect) are produced due to the low resolution of the EPIC spectra.

The variable $N_{\mathrm{w}}$ stands for the absorption model of ionized wind material, which was left as a free parameter along the whole fitting procedures. Given the high signal-to-noise ratio of the EPIC spectra (below $4 \mathrm{keV}$ ), we easily resolve single and blended spectral emission lines from OVIII, FexvII (0.825 keV), Mgxi (1.352 keV), SixiII (1.864 keV), Sixiv (2.006 keV), SixiII (2.182 keV), Sxv (2.460 keV), Sxvi (2.622 keV), ArxvII (3.139 keV), and Caxx (4.107 $\mathrm{keV})$. Because of the wide range of temperature $\left(3 \times 10^{6}\right.$ to $6.2 \times 10^{7} \mathrm{~K}$ ) needed to form these emission lines, some of them cannot be fitted by the two-temperature model (see Figure 9). Moreover, there seems to be evidence of line excess about $4.8 \mathrm{keV}$ and $6.6 \mathrm{keV}$. Unfortunately, the poor quality of the data above $4 \mathrm{keV}$ does not allow us to check for the presence of the Fexxv 6.4-6.7 keV line complex that could help us to constrain the thermal and/or non-thermal emission at the hard part of the spectrum. Such detailed study can only be developed using high resolution spectroscopic data, not published at the moment.

Solar abundances are assumed throughout the whole fitting procedure. EPIC MOS and PN fitting results appear very consistent, and our models reveal no significant differences in the quality of the fit. Both procedures are nearly equivalent within the error bars of the models. The goodness of the fit was estimated using the $\chi_{v}^{2}$ statistics, and values obtained for all EPIC instruments are quite similar. Adopted

Table 9. Parameters of the EPIC spectra at HD 93129A ${ }^{\mathrm{A}}$

\begin{tabular}{|c|c|c|c|c|c|c|c|c|}
\hline \multirow[t]{2}{*}{ Obs id. } & \multirow{2}{*}{$\begin{array}{c}N_{\mathrm{w}} \\
{\left[10^{22} \mathrm{~cm}^{-2}\right]}\end{array}$} & \multirow{2}{*}{$\begin{array}{c}\text { Mod. } 1 \\
k T_{1}[\mathrm{keV}]\end{array}$} & \multirow{2}{*}{$\begin{array}{c}\text { Norm. } 1^{\mathrm{B}} \\
{\left[10^{-3}\right]}\end{array}$} & \multirow{2}{*}{$\begin{array}{c}\text { Mod. } 2 \\
k T_{2}[\mathrm{keV}]\end{array}$} & \multirow{2}{*}{$\begin{array}{c}\text { Norm. } 2^{\mathrm{B}} \\
{\left[10^{-3}\right]}\end{array}$} & \multirow{2}{*}{$\begin{array}{l}\text { Statistic } \\
\chi_{v}^{2} / \text { d.o.f }\end{array}$} & \multicolumn{2}{|c|}{ Flux $[0.5-10.0 \mathrm{keV}]^{\mathrm{C}}$} \\
\hline & & & & & & & Abs. & Un-abs. \\
\hline $1^{D}$ & $1.08 \pm 0.12$ & $0.31 \pm 0.03$ & $6.67 \pm 0.02$ & $2.18 \pm 0.45$ & $0.44 \pm 0.01$ & 0.57 & $6.54_{5.46}^{7.02}$ & $112_{90.0}^{132.6}$ \\
\hline 2 & $0.85 \pm 0.07$ & $0.30 \pm 0.02$ & $4.75 \pm 0.01$ & $1.57 \pm 0.12$ & $0.49 \pm 0.01$ & $0.62 / 702$ & $5.79_{5.21}^{6.16}$ & $82.5_{60.1}^{94.2}$ \\
\hline 3 & $0.84 \pm 0.04$ & $0.29 \pm 0.01$ & $4.48 \pm 0.08$ & $1.42 \pm 0.04$ & $0.55 \pm 0.05$ & $0.99 / 625$ & $5.66_{5.33}^{5.88}$ & $78.0_{66.9}^{83.4}$ \\
\hline
\end{tabular}

${ }^{A}$ Results are given for MOS1, MOS2, and PN combined observations and fitted simultaneously. Quoted errors refer to $1 \sigma$ errors. The cold absorption component is frozen at $0.286 \times 10^{22} \mathrm{~cm}^{-2}$. ${ }^{\mathrm{B}}$ The normalization parameter of the model is defined as $10^{-14} /\left(4 \pi D^{2}\right) \int n_{\mathrm{e}} n_{\mathrm{H}} \mathrm{dV}$. ${ }^{\mathrm{C}}$ Flux are in units of $10^{-13} \mathrm{erg} \mathrm{cm}^{-2} \mathrm{~s}^{-1}$. D The EPIC PN camera was not used due to bad calibrated events in the PN observation. 
parameters were estimated using all EPIC spectra simultaneously.

In the $0.5-10.0 \mathrm{keV}$ range, we calculated an observed $\mathrm{X}$-ray flux of $5.66 \times 10^{-13} \mathrm{erg} \mathrm{cm}^{-2} \mathrm{~s}^{-1}$, while the corrected absorbed X-ray flux is $78.0 \times 10^{-13} \mathrm{erg} \mathrm{cm}^{-2} \mathrm{~s}^{-1}$. For a source distance of $2.5 \mathrm{kpc}$, the unabsorbed X-ray luminosity is $L_{\mathrm{X}}=4.51 \times 10^{33} \mathrm{erg} \mathrm{s}^{-1}$. According to the spectral type of HD 93129AB, its bolometric luminosity is about $1.20 \times$ $10^{40} \mathrm{erg} \mathrm{s}^{-1}$, then $L_{\mathrm{X}} / L_{\mathrm{bol}}=3.75 \times 10^{-7}$. This value agrees with the canonical $L_{\mathrm{X}} / L_{\mathrm{bol}}$ relationship for $\mathrm{O}$ type stars of the region (Albacete Colombo, Méndez, \& Morrell 2003).

\section{$6.3 X$-rays from $H D 124314$}

We found just one X-ray candidate in the HEASARC master compilation of X-ray sources within $0.05^{\circ}$ of the HD 124314 position, at $(\mathrm{RA}, \mathrm{Dec})_{\mathrm{J} 2000}=\left(14^{\mathrm{h}} 15^{\mathrm{m}} 00^{\mathrm{s}} .934\right.$, $\left.-61^{\circ} 42^{\prime} 19.91^{\prime \prime}\right)$. This source was detected by the ROSAT All-Sky Survey of Faint Sources Catalog (RASSFSC), and is named 1RXS J141500.1-614231, with a total exposure time of $13472 \mathrm{~s}$. We estimated a total count rate of $1.82 \times 10^{-3}, 0.36 \times 10^{-3}$, and $1.45 \times 10^{-3} \mathrm{cts} \mathrm{s}^{-1}$ in the total (wide) (0.11-2.4 keV), S (0.2-0.5 keV), and $\mathrm{H}(0.5-2.4$ $\mathrm{keV}$ ) energy bands, respectively. We also estimated a hardness ratio $(\mathrm{H}-\mathrm{S}) /(\mathrm{H}+\mathrm{S})$ of about $0.60 \pm 0.09$. We expect the star emits detectable X-rays; the fact that HD 124314 is a field star, combined with the positional coincidence between the star and the X-ray source lead us to assume that the X-ray source is physically related with the star.

At a distance of $1 \mathrm{kpc}$ (de Wit et al. 2004), we computed a total Galactic Hi column density of $1.84 \times 10^{22} \mathrm{~cm}^{-2}$.

With the PIMMS software we calculated an un-absorbed $\mathrm{X}$-ray flux of $2.67 \times 10^{-13} \mathrm{erg} \mathrm{cm}^{-2} \mathrm{~s}^{-1}$. The estimated ratio $\log \left(L_{\mathrm{bol}} / \mathrm{L}_{\odot}\right)=5.32$ for this star. Then, $L_{\mathrm{X}} / L_{\mathrm{bol}}$ is about $0.83 \times 10^{-7}$.

\section{$6.4 X$-rays from HD 150136}

Berghöfer et al. (1997) identified a ROSAT source of $L_{\mathrm{X}}(0.1-2.4 \mathrm{keV})=1.12 \times 10^{33} \mathrm{erg} \mathrm{s}^{-1}$, with HD 150136 , although the emission came also from HD 150135. In order to both determine the precise origin of this emission, and study the emission process, Skinner et al. (2005) performed Chandra observations towards the cluster NGC 6193, over $90000 \mathrm{~s}$. They found a soft photon energy distribution from HD 150136, interpreted as indicative of the presence of shocks in the winds.

The results of their $2 \mathrm{~T}$ model fitting $(0.35 \pm 0.04$, and $1.00 \pm 0.07 \mathrm{keV}$ ), assuming $N_{\mathrm{HI}}=4 \times 10^{21} \mathrm{~cm}^{-2}$, yielded an X-ray flux of $3.3 \times 10^{-12} \mathrm{erg} \mathrm{cm}^{-2} \mathrm{~s}^{-1}$ and $L_{\mathrm{X}}$ $=2.45 \times 10^{33} \mathrm{erg} \mathrm{s}^{-1}$ at the $(0.5-6) \mathrm{keV}$ band, obtaining $L_{\mathrm{X}} / L_{\mathrm{bol}}=3.98 \times 10^{-7}$.

The spectrum of HD 150136 presented by Skinner et al. (2005) showed broad lines. They also reported flux variability on timescales of hours, and proposed it can be produced by occultation effects from a third, and optically unseen, companion (its existence was suggested also by the optical results from Niemela \& Gamen 2005).
Table 10. X-ray luminosities for the target stars

\begin{tabular}{lccc}
\hline Star & $\begin{array}{c}\text { Energy range } \\
{[\mathrm{keV}]}\end{array}$ & $\begin{array}{c}L_{\mathrm{X}} \\
{\left[\mathrm{erg} \mathrm{s}^{-1}\right]}\end{array}$ & $\begin{array}{c}L_{\mathrm{X}} / L_{\text {bol }} \\
{\left[\times 10^{-7}\right]}\end{array}$ \\
\hline $\mathrm{CD}-47^{\circ}$ 4551 & $0.1-2.4$ & $2.73 \times 10^{31}$ & 0.12 \\
HD 93129A & $0.5-10$. & $4.51 \times 10^{33}$ & 3.80 \\
HD 124314 & $0.1-2.4$ & $6.70 \times 10^{31}$ & 0.83 \\
HD 150136 & $0.5-6.0$ & $2.45 \times 10^{33}$ & 3.99 \\
\hline
\end{tabular}

Table 10 lists the X-ray luminosity and luminosity ratio for the target stars, for comparison.

\section{Discussion}

\section{$7.1 C D-47^{\circ} 4551$ and $H D 124314$}

HD 124314 is listed as a possible spectroscopic binary, and has a visual companion. To proceed with our analysis, the binarity status of this star needs to be confirmed, and the spectral classification of the companion, derived.

$\mathrm{CD}-47^{\circ} 4551$ is not listed as a binary and it has no visual companion. Both stars/systems are currently being monitored at optical wavelengths to study their structure.

The presence of non-thermal radio emission suggests that at least part of the X-ray emission should be nonthermal, too. We found two ROSAT sources: one over $\mathrm{CD}-47^{\circ} 4551$, and another over HD 124314, which can be generated by relativistic bremsstrahlung and inverse Compton processes.

The NT spectral index of CD- $47^{\circ} 4551$ of $\alpha_{3-6 \mathrm{~cm}} \approx-1$ implies an electron distribution index of $p \approx-1$. For HD 124314 , the NT spectral index $\alpha_{\mathrm{NT}} \approx-0.7$ and $p \approx-0.4$.

The difference of the $p$ values with the one corresponding to particle acceleration in a strong adiabatic wind $(\approx-2)$ can be found by studying the conditions present at the acceleration region, and depends also on the relevance of the radiative losses. However, the difference can also be taken as an indication that the hypothesis, that the observations presented here are quasi-simultaneous, has to be revised. Really simultaneous multifrequency observations are needed instead to perform an analysis capable of explaining the radio spectra of these hot stars.

HD 124314 lies inside the location probability contours of the EGRET source 3EG J1410-6147 (Romero, Benaglia, $\&$ Torres 1999), one of the few high-energy sources with $E>10 \mathrm{GeV}$. The counterpart of this EGRET source has been proposed to be the SNR G312.4-0.4 (Torres et al. 2003); nevertheless, a composite origin cannot be ruled out and high energy emission from the stellar system can make a contribution.

\subsection{HD 93129A}

From the latest optical and radio studies on the stellar system HD 93129A (Nelan et al. 2004; Benaglia \& Koribalski 2004) an approximate description of the stellar and orbital parameters can be addressed. The HST data showed a binary separation of $55 \mathrm{mas}$, or $140 \mathrm{AU}$, at $2.5 \mathrm{kpc}$. A windmomentum ratio $\eta=\left(\begin{array}{ll}M_{2} & v_{\infty, 2}\end{array}\right) /\left(M_{1} v_{\infty, 1}\right)=0.14$ is 
derived, where ' 1 ' stands for the primary star and ' 2 ' for the secondary star parameters. The derivation is based on massloss rates of $2.3 \times 10^{-5} \mathrm{M}_{\odot} \mathrm{yr}^{-1}$ and $0.3 \times 10^{-5} \mathrm{M}_{\odot} \mathrm{yr}^{-1}$, and wind velocities of $3200 \mathrm{~km} \mathrm{~s}^{-1}$ and $3000 \mathrm{~km} \mathrm{~s}^{-1}$ for the two stars (Repolust, Puls, \& Herrero 2004; Benaglia \& Koribalski 2004). These results place the colliding-wind region 15 mas from the companion star, and lead to an estimated CWR size of $\pi r_{2} \sim 50$ mas (Eichler \& Usov 1993).

The magnitude of the magnetic field as a function of distance, in wide systems, is related to the stellar surface magnetic field $B_{*}$ (Eichler \& Usov 1993) by

$$
B(r)=B_{*} \frac{v_{\mathrm{rot}}}{v_{\infty}} \frac{R_{*}^{2}}{r_{\mathrm{A}} r},
$$

where $R *$ is the stellar radius, $r_{\mathrm{A}}$ the Alfvén radius, and $v_{\text {rot }}$ the surface rotation velocity. For early-type stars, $v_{\text {rot }} \approx$ $(0.1-0.2) v_{\infty}$ and $r_{\mathrm{A}} \approx(1-3) R_{*}$.

Only in very few cases a value of the stellar magnetic field could be derived: Donati et al. (2001, 2002) measured $B_{*}$ for two early-type stars, $\beta$ Cephei (B1 IV) and $\theta^{1}$ Ori $\mathrm{C}(\mathrm{O} 4-6 \mathrm{~V})$, and obtained the values $360 \pm 30 \mathrm{G}$ and $1100 \pm 100 \mathrm{G}$, respectively. Theoretical values are about a few hundred Gauss.

Observations at the radio range can give a hint on stellar magnetic fields. When non-thermal radio emission is detected and detached from the thermal flux, an equipartition magnetic field at the CWR can be derived following Miley (1980). The following approximated expression for minimum energy conditions can be used:

$$
B_{\mathrm{eq}}[\mathrm{mG}] \approx 100\left[\frac{1}{\theta^{3} d} \frac{1+\chi}{f} \frac{S_{x}^{\mathrm{NT}}}{v_{x}^{\alpha_{\mathrm{NT}}}} \frac{v_{2}^{\alpha^{\prime}}-v_{1}^{\alpha^{\prime}}}{\alpha^{\prime}}\right]^{2 / 7} .
$$

Here $\theta$ is the CWR size [mas], $\alpha^{\prime}=\alpha^{\mathrm{NT}}+0.5, d[\mathrm{kpc}], \chi$ is the energy ratio between heavy particles and electrons, $f$ is the filling factor of the emitting region, $S_{x}$ is the flux density [mJy] of the region at frequency $v_{x}[\mathrm{GHz}]$, and $v_{1}$ $[\mathrm{GHz}]$ and $v_{2}[\mathrm{GHz}]$ are the upper and lower cut-off frequencies presumed for the radio spectrum. We have assumed that the energy of particles and electrons is the same and a filling factor of 1 .

By means of the ATCA data and a non-radio derived mass loss rate value, we determined a non-thermal spectral index $\alpha_{3-6 \mathrm{~cm}}^{\mathrm{NT}} \approx-1.5$ (see Section 5). There is clear evidence from the spectrum that non-thermal emission is present at all wavelengths. For $\theta \approx 50$ mas, an equipartition field at the emitting region of $B_{\mathrm{CWR}} \approx 10 \mathrm{mG}$ is computed, using $x=$ $8.64 \mathrm{GHz}$ in Eqn (4), and Table $5^{5}$. A corresponding (minimum) stellar magnetic field of $B * \sim 50 \mathrm{G}$ is derived, by assuming $v_{\text {rot }}=0.2 v_{\infty}, r_{\mathrm{A}}=3 R_{*}$, and $R_{*}=20 \mathrm{R}_{\odot}$ (Martins, Schaerer, \& Hillier 2005).

For this particular $B$-field, we can evaluate the frequencies below which the RTe and SSA are relevant. The electron density can be expressed in terms of the mass

\footnotetext{
${ }^{5}$ We note that the last value is relevant as an order of magnitude. A different filling factor of, lets say, $f=0.1$, or a CWR size value halved, would double the value of $B_{\mathrm{CWR}}$.
}

loss rate of the primary star, as $n_{\mathrm{e}}=\dot{M}_{1} /\left(4 \pi r_{1} v_{\infty 1} \mu_{1} m_{\mathrm{H}}\right)$, yielding $n_{\mathrm{e}} \sim 6 \times 10^{4} \mathrm{~cm}^{-3}$ and then $v_{\mathrm{RTe}} \sim 120 \mathrm{MHz}$.

From Eqn. (2), $v_{\mathrm{SSA}} \sim 25 \mathrm{MHz}$. Even if the unabsorbed flux at $1.4 \mathrm{GHz}$ is considered higher than the measured flux (i.e. $\left.5 S_{20 \mathrm{~cm}}\right) v_{\mathrm{SSA}} \sim 50 \mathrm{MHz}$. With the parameters set adopted, if the turnover at $1.4 \mathrm{GHz}$ is ascribed to SSA, the equipartition magnetic field at the CWR would be too high to represent a real scenario.

The non-thermal spectral index $\alpha_{3-6 \mathrm{~cm}}^{\mathrm{NT}}=-1.5$, implies an electron distribution with $p=-4$. Explanations of this high value can be found (a) if the injected spectrum is sufficiently steep or (b) if the radio emission is produced by a population of electrons with Lorentz factors above $\gamma_{b}$. An estimate of $\gamma_{b}$ leads to compute the radio frequency at which the spectral index change from $\left(\alpha^{\mathrm{NT}}\right)$ to $\left(\alpha^{\mathrm{NT}}+0.5\right)$ in $v_{\mathrm{b}}>10^{6} k^{-1} \mathrm{GHz}$. A $k$ value to make $v_{\mathrm{b}}$ in the $\mathrm{GHz}$ range has no physical meaning for relativistic particles.

The maximum Lorentz factor attained by the electrons at the CWR, computed as in Benaglia \& Romero (2003), is $\gamma_{\max }=1.6 \times 10^{5}$. This corresponds to a maximum synchrotron frequency of $v=1 \times 10^{6} \mathrm{GHz}$, and a cutoff energy for the IC photons of $E \approx 5 \mathrm{GeV}$.

\section{Conclusions}

We measured the spectrum of HD 93129A from 1.4 to 24.5 $\mathrm{GHz}$ and found the flux decreases with frequency. The negative spectral indices, and the high flux values detected (up to $9.4 \mathrm{mJy}$ ) for an $\mathrm{O}$ star, are consistent with synchrotron emission from a CWR. An X-ray source coincident in position with the star was found from XMM archive data analysis, and a two temperature spectrum could be fitted which accounts for the many X-ray spectral lines present. The source shows a high $L_{X} / L_{\text {bol }}$ ratio of $3.8 \times 10^{-7}$, characteristic of OB binaries. The (low) quality of the data above $4 \mathrm{keV}$ prevented us to explore the presence of Fe lines considered as a signature of colliding winds. The hard emission found by Evans et al. (2004) also favours a CWR origin. The intense NT radio emission suggests that the system could be, in principle, detectable with forthcoming high-sensitivity gamma ray telescopes.

CD-47 4551 and HD 124314 are radio non-thermal emitters, and the spectra show a turnover from 1.4 to 8.64 $\mathrm{GHz}$. Their detected fluxes were strongly contaminated with non-thermal emission. Precise (optical) information about the structure of their systems, lacking at the moment, is crucial to reveal the presence of close early-type companions and wind colliding regions, in order to perform a quantitative radio study towards these stars. Both stars are coincident with ROSAT sources, identified here with the stars, and for which we estimated the corresponding luminosity. The $L_{X} / L_{\text {bol }}$ ratios obtained for them $\left(\sim 10^{-8}\right)$ appear low for strong wind collisions: We found no relevant excess when these fluxes are compared with those corresponding to single stars.

HD 150136 is in a multiple system, with at least three early-type stars. Its radio emission also shows important nonthermal contamination, up to $8.64 \mathrm{GHz}$, though HD 150136 is seen as a point source. To account for the contributions in 
its radio spectrum, very long-baseline, multifrequency radio observations (separated at most by a few hours) are fundamental. The extensive X-ray investigation by Skinner et al. (2005) permits to derive a high $L_{X} / L_{\text {bol }}$ ratio of $4 \times 10^{-7}$, characteristic of heated gas at a wind collision event.

The picture of these four interesting southern NT OB systems could benefit if at least some of the following studies are pursued: (a) simultaneous radio observations at several frequencies and at higher angular resolution to image and resolve the wind region, (b) optical studies looking for companions, both to discover the structure of the stellar systems and to classify the components, (c) dedicated high angular resolution X-ray observations including spectroscopy, and (d) high-resolution/sensitivity gammaray observations.

\section{Acknowledgments}

P.B. thanks G. E. Romero for useful discussions, JBR, the ATNF staff at Marsfield and Narrabri for constant help, and N. R. Walborn for a critical reading of the manuscript. J.F.A.C. acknowledges support by the Marie Curie Fellowship Contract No. MTKD-CT-2004-002769 of the project 'The Influence of Stellar High Energy Radiation on Planetary Atmospheres', and the host institution INAF (Osservatorio Astronomico di Palermo, OAPA). This research has been supported by the Argentine agency ANPCyT through grant PICT 03-13291.

\section{References}

Albacete Colombo, J. F., Méndez, M., \& Morrel, N. I. 2003, MNRAS, 346, 704

Bell, A. R. 1978, MNRAS, 182, 147

Benaglia, P., Cappa, C. E., \& Koribalski, B. S. 2001, A\&A, 372, 952 Benaglia, P., \& Koribalski, B. 2004, A\&A, 416, 171

Benaglia, P., \& Koribalski, B. 2006, in Massive Stars in Interacting Binaries (Eds. Moffat, A. F. J., \& St Louis, N.) in press.

Benaglia, P., \& Romero, G. E. 2003, A\&A, 399, 1121

Berghofer, T. W., Schmitt, J. H. M. M., Danner, R., \& Cassinelli, J. P. 1997, A\&A, 322, 167

Bieging, J. H., Abbott, D. C., \& Churchwell, E. B. 1989, ApJ, 340, 518 Blomme, R., van Loo, S., De Becker, M., et al. 2005, A\&A, 436, 1033 Chen, W., \& White, R. L. 1991, ApJ, 381, L63

Chen, W., \& White, R. L. 1994, Ap\&SS, 221, 259

Chlebowski, T., \& Garmany, C. D. 1991, ApJ, 368, 241

Cohen, D. H., de Messieres, G. E., MacFarlane, J. J. et al. 2003, ApJ, 586, 495

Contreras, M. E., Rodriguez, L. F., Tapia, M., et al. 1997, ApJ, 488, L153

De Becker, M., Rauw, G., Blomme, R., et al. 2004, A\&A, 420, 1061

DeGioia-Eastwood, K., Throop, H., Walker, G., \& Cudworth, K. M. 2001, ApJ, 549, 578

de Wit, W. J., Testi, L., Palla, F., et al. 2004, A\&A, 425, 937

Donati, J.-F., Babel, J., Harries, T. J., et al. 2002, MNRAS, 333, 55

Donati, J.-F., Wade, G. A., Babel, J., et al. 2001, MNRAS, 326, 1265

Done, C., Mulchaey, J. S., Mushotzky, R. F., \& Arnaud, K. A. 1992, ApJ, 395, 275

Dougherty, S. M., Beasley, A. J., Coker, R. F., \& Corcoran, M. F. 2005, ApJ, 623, 447

Dougherty, S. M., Pittard, J. M., Kasian, L., et al. 2003, A\&A, 409, 217
Dougherty, S. M., Williams, P. M., van der Hucht, K. A., et al. 1996, MNRAS, 280, 963

Eichler, D., \& Usov, V. 1993, ApJ, 402, 271

Evans, N. R., Schlegel, E. M., Waldron, W. L., et al. 2004, ApJ, 612, 1065

Evans, N. R., Seward, F. D., Krauss, M. I, et al. 2003, ApJ, 589, 509

Feast, M. W., Thackeray, A. D., \& Wesselink, A. J. 1954, MmRAS, 67, 51

Herbst, W., \& Havlen, R. J. 1977, A\&AS, 30, 279

Howarth, I. D., Siebert, K. W., Hussain, G. A. J., \& Prinja, R. K. 1997, MNRAS, 284, 265

Jardine, M., Collier Cameron, A., Donati, J.-F., \& Pointer, G. R. 2001, MNRAS, 324, 201

Kahn, S. M., Leutenegger, M. A., Cottam, J., et al. 2001, A\&A, 365, L312

Longair, M. S. 1997, High Energy Astrophysics, Vol. 2, p. 281 (Cambridge: Cambridge University Press)

Lucy, L. B., \& White, R. L. 1980, ApJ, 241, 300

Maíz-Apellániz, J., Úbeda, L., Walborn, N.R., \& Nelan E.P. 2006, in Resolved Stellar Populations, ASP Conf. Ser. (Eds VallsGabaud, D., \& Chávez, M.) in press (San Francisco: ASP)

Maíz-Apellániz, J., Walborn, N. R., Galué, H. A., \& Wei, L. H. 2004, ApJS, 151, 103

Martins, F., Schaerer, D., \& Hillier, D. J. 2005, A\&A, 436, 1049

Mason, B., Gies, D. R., Hartkopf, W. I., et al. 1998, AJ, 115, 821

Miley, G. K. 1980, ARA\&A, 18, 165

Moran, J. P., Davis, R. J., Spencer, R. E., et al. 1989, Nat, 340, 449

Morrison, R., \& McCammon, D. 1983, ApJ, 270, 119

Nelan, E. P., Walborn, N. R., Wallace, D. J., et al. 2004, ApJ, 128, 323

Niemela, V. S., \& Gamen, R. C. 2005, MNRAS, 356, 974

Niemela, V. S., Gamen, R. C., Solivella, G., Benaglia, P., et al. 2006, submitted

O’Connor, E. P., Dougherty, S. M., Pittard, J. M, \& Williams, P. M. 2005, in Massive Stars and High-Energy Emission in OB Associations, Proc JENAM (Eds. Rauw, G., Nazé, Y., Blomme, R., \& Gosset, E.) p. 81

Pachlolczyk, A. G. 1970, Radio Astrophysics (San Francisco: Freeman)

Pollock, A. M. T. 1987, A\&A, 171, 135

Prinja, R. K., Barlow, M. J., \& Howarth, I. D. 1990, ApJ, 361, 607

Rauw, G., Sana, H., Gosset, E. et al. 2005, in Massive Stars and High-Energy Emission in OB Associations, Proc JENAM (Eds. Rauw, G., Nazé, Y., Blomme, R., \& Gosset, E.), p. 85

Repolust, T., Puls, J., \& Herrero, A. 2004, A\&A, 415, 349

Romero, G. E., Benaglia, P., \& Torres, D. F. 1999, A\&A, 348, 868

Shull, J. M., \& van Steenberg, M. E. 1985, ApJ, 294, 599

Skinner, S. L., Itoh, M., Nagase, F., \& Zhekov, S. A. 1999, ApJ, 524, 394

Skinner, S. L., Zhekov, S. A., Palla, F., \& Barbosa, C. L. D. R. 2005, MNRAS, 361, 191

Taresch, G., Kudritzki, R. P., Hurwitz, M., et al. 1997, A\&A, 321, 531

Torres, D. F., Romero, G. E., Dame, T. M., Combi, J. A., \& Butt, Y. M. 2003, PhR, 382, 303

van Loo, S. 2005, Ph.D. Thesis (Leuven: University of Leuven)

Vink, J. S., de Koter, A., \& Lamers, H. J. G. L. M. 2000, A\&A, 362,295

Walborn, N. R. 1973, ApJ, 179, 517

Walborn, N. R. 1982, AJ, 87, 1300

Walborn, N. R. 1995, RMxAA, 2, 51

Walborn, N. R., Howarth, I. D., Lennon, D. J., et al. 2002, AJ, 123,2754

Wegner, W. 1994, MNRAS, 270, 229

White, R. L. 1985, ApJ, 289, 698

Williams, P. J. S. 1963 , Nat 200, 56

Wright, A. E., \& Barlow, M. J. 1975, MNRAS, 170, 41

Yamaguchi, N., Mizuno, N., Moriguchi, Y. et al. 1999, PASJ, 51, 765 